\title{
Lnc Tmem235 promotes repair of early steroid- induced osteonecrosis of the femoral head by regulating miR-34a-3p/BIRC5 to inhibit hypoxia- induced apoptosis of bone-marrow mesenchymal stem cells
}

Fei Zhang

Affliated Hospital of Guizhou Medical University

Wuxun Peng ( 903463644@qq.com )

The Affiliated Hospital of Guizhou Medical University

\section{Tao Wang}

Guiyang Medical University

Jian Zhang

Wentao Dong

The Affliated Hospital of Guizhou Medical University

\section{Chuan Wang}

Guizhou Medical University

\section{Zhihong Xie}

Guiyang Medical University

\section{Yanglin Yan}

Guizhou Medical University

\section{Article}

Keywords: Bone-marrow mesenchymal stem cells (BMSCs), Lnc Tmem235, osteonecrosis of the femoral head (SONFH)

Posted Date: July 13th, 2021

DOI: https://doi.org/10.21203/rs.3.rs-95235/v1

License: (c) (i) This work is licensed under a Creative Commons Attribution 4.0 International License. Read Full License 


\section{Lnc Tmem 235 promotes repair of early steroid-induced osteonecrosis}

of the femoral head by regulating miR-34a-3p/BIRC5 to inhibit

\section{hypoxia-induced apoptosis of bone-marrow mesenchymal stem cells}

\section{Abstract}

Bone-marrow mesenchymal stem cells (BMSCs) have been used in the treatment of early steroid-induced osteonecrosis of the femoral head (SONFH). However, the hypoxic microenvironment in the osteonecrotic area leads to hypoxia-induced apoptosis of transplanted BMSCs, which limits their efficacy. Therefore, approaches that inhibit hypoxia-induced apoptosis of BMSCs are promising for augmenting the efficacy of BMSC transplantations. Our present study found that under hypoxia, expression of the long non-coding RNA (Lnc), transmembrane protein 235 (Tmem235), was down-regulated, expression of Bcl-2-associated $\mathrm{X}$ protein was up-regulated, expression of B-cell lymphoma-2 protein was down-regulated, and the apoptotic rate of BMSCs was over 70\%. However, overexpression of Lnc Tmem235 reversed hypoxia-induced apoptosis of BMSCs and promoted their survival. These results demonstrated that Lnc Tmem235 effectively inhibited hypoxia-induced apoptosis of BMSCs. Mechanistically, we found that Lnc Tmem235 exhibited competitive binding to miR-34a-3p with BIRC5 mRNA, which is an inhibitor of apoptosis; this competitive binding relieved the silencing effect of miR-34a-3p on BIRC5 mRNA to ultimately inhibit hypoxia-induced apoptosis of BMSCs by promoting the expression of BIRC5. Furthermore, we co-cultured BMSCs overexpressing Lnc Tmem235 with xenogeneic antigen-extracted cancellous bone to construct tissue-engineered bone to repair a model of early SONFH in vitro. The results showed that overexpression of Lnc Tmem 235 effectively reduced apoptosis of BMSCs in the hypoxic microenvironment of osteonecrosis and improved the effect of BMSC transplantation. Taken together, our findings elucidate that Lnc Tmem235 inhibited hypoxia-induced apoptosis of BMSCs by regulating the miR-34a-3p/BIRC5 axis, thus improving the transplantation efficacy of BMSCs for treating early SONFH. 


\section{Introduction}

The incidence of steroid-induced osteonecrosis of the femoral head (SONFH) is increasing year by year and accounts for $25 \%-51 \%$ of the total number of cases of osteonecrosis of the femoral head, ranking first in non-traumatic osteonecrosis of the femoral head [1-2]. If not treated in time, the collapse rate of osteonecrosis of the femoral head within two years is more than $80 \%$, and the disability rate is markedly high. Therefore, early treatment is particularly important [2-4]. Transplantation of bone-marrow mesenchymal stem cells (BMSCs) represents a novel method for treating early SONFH [5-6]. However, the oxygen concentration in the necrotic area of the femoral head in SONFH cases is usually lower than $1 \%$, and a local hypoxic microenvironment is formed, which can lead to hypoxia-induced apoptosis of transplanted BMSCs, limiting their capacity for osteogenic repair [7-14]. Therefore, identification and development of methods for inhibiting hypoxia-induced apoptosis of BMSCs are key to further improving the efficacy of BMSC transplantations. At present, methods to inhibit apoptosis of BMSCs mainly include drug pretreatments, improvement of mitochondrial function, clearance of excessive reactive oxygen species (ROS), and inhibition of apoptosis-related proteins; however, the efficacies of these methods remain unsatisfactory [15-18]. Therefore, it is of great practical significance to identify novel targets and develop new methods for inhibiting hypoxia-induced apoptosis of BMSCs and improving the efficacy of BMSC transplantations.

Long non-coding RNAs (LncRNAs) are non-coding RNA molecules with transcript lengths between $200 \mathrm{nt}-100 \mathrm{~kb}$. Upon their initial discovery, LncRNAs were initially considered as noise in the process of gene transcription and were not thought to have any biological function. However, recent studies have shown that LncRNAs can regulate gene expression through DNA methylation, histone modification, control of transcription factors, regulation of RNA stability, and subcellular localization at pre-transcriptional, transcriptional, and post-transcriptional levels, thus interfering with a variety of physiological activities, such as apoptosis 
[19-22]. For example, Lnc TNRC6C-AS1 inhibits apoptosis of thyroid cancer cells by promoting methylation of STK4 [23]. Additionally, Lnc TCF7 recruits the transcription factor complex, SWI/SNF, into the TCF7 promoter region to regulate its expression, thus promoting the self-renewal and proliferation of liver cancer stem cells [24]. Furthermore, Lnc H19 regulates intracellular localization of HIF-1 $\alpha$ at the posttranslational level, thus interfering with apoptosis of smooth muscle cells [25]. In addition, LncRNA-regulated gene expression is closely related to microRNAs (miRNAs) [26-27].

miRNAs are small non-coding single-stranded RNAs that can bind to the untranslated region (UTR) at the $3^{\prime}$ end of a target mRNA (mRNA 3'UTR) to inhibit mRNA translation and cause gene silencing. Studies have shown that LncRNAs can act as competitive endogenous RNAs (ceRNAs) to competitively bind miRNAs with the mRNA of the target gene, thereby relieving the silencing effect of miRNAs on the target gene to ultimately promote expression of the target gene and interfere with various physiological activities such as apoptosis [26-27]. For example, Lnc CARL can be used as a sponge of endogenous miR-539 to regulate the expression of PHB2, thereby intervening in cardiomyocyte apoptosis [28]. As another example, Lnc LOXL1-AS1 can be used as a ceRNA to compete with RAP1B mRNA for binding with miR-28-5p to promote RAP1B expression, ultimately inhibiting apoptosis of endometrial cancer cells [29]. However, little is known about the role of LncRNAs and miRNAs in the process of hypoxia-induced apoptosis of BMSCs. Hence, it is not clear whether novel targets or methods can be identified for inhibiting hypoxia-induced apoptosis of BMSCs and whether inhibition of hypoxia-induced apoptosis of BMSCs may further improve the transplantation efficacy of BMSCs for treating early SONFH.

In the present study, we explored the effects and mechanisms of the interaction between Lnc Tmem235 and miR-34a-3p on hypoxia-induced apoptosis of BMSCs. Furthermore, we evaluated the effects of inhibiting hypoxia-induced apoptosis of BMSCs in terms of treating early SONFH. Collectively, our findings may contribute to the identification of novel targets and development of methods for inhibiting 
hypoxia-induced apoptosis of BMSCs and improving the efficacy of BMSC transplantations.

\section{Results}

1. Expression of Lnc Tmem235 is down-regulated in the process of hypoxia-induced apoptosis of BMSCs

To investigate the effect of hypoxia on apoptosis of BMSCs, we subjected BMSCs to hypoxia $(0 \% \mathrm{O} 2,95 \% \mathrm{~N} 2$, and $5 \% \mathrm{CO} 2)$ for $48 \mathrm{~h}$. The results showed that after BMSCs were exposed to hypoxia, the mitochondrial membrane potential and ATP levels were decreased (Figure 1A-C), while ROS levels were increased (Figure 1D-E); furthermore, the expression of B-cell lymphoma-2 (Bcl-2) was down-regulated, Caspase-3 (CASP-3) and Bcl-2-related X (Bax) were up-regulated (Figure $1 \mathrm{H}-\mathrm{K}$ ), and a large number of BMSCs were apoptotic (Figure 1F-G).

LncRNAs can regulate gene expression in many different ways and play important roles in the regulation of apoptosis [19-22]. Hence, we next determined which LncRNAs were related to hypoxia-induced apoptosis of BMSCs. First, we cultured BMSCs under hypoxia $(0 \% \mathrm{O} 2,95 \% \mathrm{~N} 2$ and $5 \% \mathrm{CO} 2)$ for $48 \mathrm{~h}$, and then searched for hypoxia-reactive LncRNAs and mRNAs via an LncRNA chip. The results showed that 99 LncRNAs were up-regulated, 183 LncRNAs were down-regulated (Figure 1L-N), 422 mRNAs were up-regulated, and 1104 mRNAs were down-regulated (Fold change $>2, P<0.05$ ) (Figure $1 \mathrm{O}-\mathrm{Q}$ ). Then, gene ontology (GO) and Kyoto Encyclopedia of Genes and Genomes (KEGG) pathway analyses were performed on these differentially-expressed mRNAs to determine apoptosis-related mRNAs (Fold change $>4, P<0.01$ ). We then analyzed mRNA/LncRNA co-expression (abs $\geqslant 0.98, \mathrm{p}$-value $\leqslant 0.01$, FDR $\leqslant 1$ ) and corresponding gene-location relationships (distance $<200 \mathrm{~kb}$ ). We identified two apoptosis-related candidate LncRNAs: Tmem235 and LOC102553514 (Figure 1R-S). Next, we used quantitative real-time PCR (qPCR) to verify the expression of candidate LncRNAs in our BMSC hypoxia model, and we found that the results were consistent with our microarray data (Figure 1T). Among them, the expression of Lnc 
117 Tmem235 was significantly down-regulated, and its expression was continuously

118 down-regulated with the aggravation of hypoxia and the concomitantly-increased 119 apoptotic rate, while the expression of Lnc LOC102553514 was not related to the 120 degree of hypoxia (Figure 1U-W). These results suggest that Lnc Tmem235 may be 121 related to hypoxia-induced apoptosis of BMSCs.

\section{Lnc Tmem235 inhibits hypoxia-induced apoptosis of BMSCs}

To further study the effects of Lnc Tmem235 on hypoxia-induced apoptosis of BMSCs, we transfected BMSCs with an Lnc Tmem235 overexpression lentivirus (Lv-Lnc Tmem235) in order to overexpress Lnc Tmem235 (Figure 2A). Then, BMSCs were treated with hypoxia $(0 \% \mathrm{O} 2,95 \% \mathrm{~N} 2$, and $5 \% \mathrm{CO} 2)$ for $48 \mathrm{~h}$. The results showed that under hypoxia, the expression levels of Lnc Tmem235 and Bcl-2 were down-regulated while the expression levels of Bax and CASP-3 were up-regulated (Figure 2B-E), and the BMSC apoptotic rate was more than $70 \%$ (Figure 2F-I). However, overexpression of Lnc Tmem235 reversed the above results, as it significantly reduced the apoptotic rate of BMSCs and promoted the survival of BMSCs under hypoxia (Figure 2). These findings suggest that Lnc Tmem235 inhibited hypoxia-induced apoptosis of BMSCs. However, the mechanism by which Lnc Tmem235 inhibited hypoxia-induced apoptosis in BMSCs remains unclear.

\section{Lnc Tmem235 inhibits hypoxia-induced apoptosis of BMSCs by regulating} BIRC5

To elucidate the mechanism by which Lnc Tmem 235 inhibited hypoxia-induced apoptosis of BMSCs, we treated BMSCs with overexpressing Lnc Tmem235 (BMSCs-Lnc Tmem235) and subjected them to hypoxia $(0 \% \mathrm{O} 2,95 \% \mathrm{~N} 2$ and, 5\% $\mathrm{CO} 2)$ for $48 \mathrm{~h}$. Then, we detected the gene expression profile of BMSCs-Lnc Tmem 235 by microarray to screen the downstream genes that may be regulated by Lnc Tmem235. The results showed that 156 mRNAs were up-regulated and 659 mRNAs were down-regulated (Fold change $>2, P<0.05$ ), among which the expression of bacterial inhibitor of apoptosis (IAP) repeat-containing 5 (BIRC5) was significantly up-regulated (Figure 3A). Then, we used qPCR to verify the microarray data, and the results showed that Lnc Tmem235 promoted the expression of BIRC5 in 
BMSCs under hypoxia (Figure 3B-C). BIRC5, as an apoptotic inhibitor, can directly inhibit the activities of CASP-3 and CASP-9, thereby blocking apoptosis [30-33]. Therefore, we speculated that Lnc Tmem235 may have inhibited hypoxia-induced apoptosis of BMSCs by regulating the expression of BIRC5.

We next determined whether Lnc Tmem235 inhibited hypoxia-induced apoptosis of BMSCs via regulation of BIRC5. We first transfected BMSCs with Lv-Lnc Tmem235 to make BMSCs up-regulate Lnc Tmem235 (Figure 3D). Then, we subjected BMSCs to hypoxia $(0 \% \mathrm{O} 2,95 \% \mathrm{~N} 2$, and $5 \% \mathrm{CO} 2)$ for $48 \mathrm{~h}$. We detected that the activities of CASP-3 and CASP-9 and hypoxia-induced apoptosis of BMSCs were decreased significantly after up-regulation of Lnc Tmem235 (Figure 3H-L). Then, on the basis of up-regulating Lnc Tmem235, we further transfected BMSCs with a BIRC5 interference lentivirus, which resulted in down-regulation of BIRC5 expression (Figure 3E-G). The results showed that down-regulation of BIRC5 significantly weakened the anti-apoptotic effect of Lnc Tmem235 (Figure 3H-L). On the contrary, we transfected BMSCs with Lv-Sh-Lnc Tmem235, which significantly decreased the expression of Lnc Tmem235 (Figure 3M), and the activities of CASP-3 and CASP-9 and hypoxia-induced apoptosis of BMSCs were increased significantly after Lnc Tmem235 was down-regulated (Figure 3Q-X). Then, on the basis of down-regulating Lnc Tmem235, we further transfected BMSCs with a BIRC5 overexpression lentivirus (Lv-BIRC5), which resulted in up-regulation of BIRC5 expression (Figure 3N-P). The results showed that up-regulation of BIRC5 effectively ameliorated hypoxia-induced apoptosis of BMSCs (Figure 3Q-X). These results confirmed that Lnc Tmem235 inhibited hypoxia-induced apoptosis of BMSCs by regulating BIRC5 expression. However, it remains unclear how LncTmem235 regulates the expression of BIRC5.

\section{Lnc Tmem235 regulates BIRC5 expression by targeting miR-34a-3p}

Studies have shown that LncRNA can competitively bind to miRNAs with target gene mRNAs, thus releasing the silencing effect of miRNAs on target genes to ultimately promote the expression of target genes [34]. Hence, we next investigated whether Lnc Tmem235 regulated the expression of BIRC5 through the above 
mechanism. We used bioinformatic tools (mirdb and rnahybrid) to predict possible binding miRNAs of Lnc Tmem235 and untranslated regions at the 3' end of BIRC5 mRNA (BIRC5 mRNA 3'UTR). The results showed that miR-34a-3p could bind to Lnc Tmem235 and BIRC5 mRNA 3'UTR simultaneously (Figure 4A), and that it had the same binding site on miR-34a-3p. Thus, Lnc Tmem235 and BIRC5 mRNA satisfied conditions for competitive binding to miR-34a-3p (Figure 4B). Hence, we speculated that Lnc Tmem235 may competitively bind miR-34a-3p with BIRC5 mRNA, thus releasing the silencing effect of miR-34a-3p on BIRC5 mRNA to ultimately promote the expression of BIRC5.

We next determined whether miR-34a-3p could combine with Lnc Tmem235 and BIRC5 mRNA simultaneously. First, we overexpressed miR-34a-3p in BMSCs (Figure 4C), and detected the enrichment of Lnc Tmem235 and BIRC5 mRNA to miRNA ribonucleoprotein complexes (miRNPs) via RNA-binding protein immunoprecipitation (RIP). The results showed that overexpression of miR-34a-3p significantly increased the enrichment of Lnc Tmem235 and BIRC5 mRNA to miRNPs (Figure 4D-F). Then, we inserted the cDNA of Lnc Tmem235 and BIRC5 mRNA 3'UTR downstream of the luciferase (Luc) reporter gene to construct overexpression lentiviruses (Lv-Luc-Lnc Tmem235 and Lv-Luc-BIRC5 3'UTR) to transfect BMSCs. Meanwhile, BMSCs were transfected with an miR-34a-3p overexpression lentivirus (Lv-miR-34a-3p). Our luciferase-reporter assay showed that luciferase activity was decreased significantly after miR-34a-3p was up-regulated (Figure 4G-H). When we mutated the predicted binding sites of Lnc Tmem235 or BIRC5 mRNA 3'UTR on miR-34a-3p (Lnc Tmem235 MUT and BIRC5 3'UTR MUT), we used the same method to construct overexpression lentiviruses (Lv-Luc-Lnc Tmem235 MUT and Lv-Luc-BIRC5 3'UTR MUT) to transfect BMSCs. At this time, our luciferase-reporter assay showed that up-regulation of miR-34a-3p expression had no significant effect on luciferase activity (Figure 4G-H). These results indicate that miR-34a-3p can bind to the predicted sites on Lnc Tmem235 or BIRC5 mRNA.

We next investigated whether Lnc Tmem 235 can competitively bind miR-34a-3p 
with BIRC5 mRNA to regulate the expression of BIRC5. We first transfected BMSCs

with Lv-BIRC5 to over-express BIRC5, and then transfected BMSCs with Lv-miR-34a-3p to up-regulate the expression of miR-34a-3p (Figure 4I). Finally, the enrichment of BIRC5 mRNA into miRNPs was detected by RIP, and the mRNA and protein levels of BIRC5 were detected by qPCR and western blotting, respectively. The results showed that BIRC5 mRNA was significantly enriched in miRNPs, whereas the expression of BIRC5 was down-regulated (Figure 4L-O). Furthermore, on the basis of up-regulating miR-34a-3p, we further up-regulated Lnc Tmem235 (Figure 4J); the results showed that Lnc Tmem235 was significantly enriched in miRNPs, the enrichment of BIRC5 mRNA to miRNPs decreased, and BIRC5 expression was up-regulated (Figure $4 \mathrm{~K}-\mathrm{O}$ ).

The above results confirmed that Lnc Tmem235 could be used as a ceRNA to competitively bind miR-34a-3p with BIRC5 mRNA, thereby relieving the silencing effect of miR-34a-3p on BIRC5 mRNA and ultimately promoting the expression of BIRC5.

\section{Lnc Tmem235 inhibits hypoxia-induced apoptosis of BMSCs by regulating} miR-34a-3p/BIRC5.

We next determined whether Lnc Tmem235 inhibited hypoxia-induced apoptosis of BMSCs by regulating miR-34a-3p/BIRC5. We transfected BMSCs with Lv-LncTmem235 to overexpress LncTmem235 in BMSCs (Figure 5A), and then subjected BMSCs to hypoxia $(0 \% \mathrm{O} 2,95 \% \mathrm{~N} 2$, and $5 \% \mathrm{CO} 2)$ for $48 \mathrm{~h}$. Compared with that of the Lv-EGFP group, the expression of BIRC5 in the Lv-Lnc Tmem235 group was up-regulated (Figure $5 \mathrm{C}-\mathrm{E}$ ), and apoptosis was significantly reduced (Figure 5F-I). When we up-regulated the expression of miR-34a-3p on the basis of overexpression of Lnc Tmem235 (Figure 5B), the content of BIRC5 was decreased and apoptosis was increased. The results showed that miR-34a-3p blocked the anti-apoptotic effect of Lnc Tmem235 (Figure 5C-I). Subsequently, on the basis of overexpression of Lnc Tmem235 and miR-34a-3p, we up-regulated the expression of BIRC5. Following this manipulation, apoptosis was reduced, revealing that up-regulation of BIRC5 rescued BMSCs from hypoxia-induced apoptosis (Figure 
5C-I). These results confirmed that LncTmem235 inhibited hypoxia-induced apoptosis of BMSCs by regulating the miR-34a-3p/BIRC5 axis.

6. Lnc Tmem235 inhibits hypoxia-induced apoptosis of BMSCs and promotes repair of early SONFH (40 mg/kg/d) to establish an early SONFH model (Figure 6A). At the sixth week of modeling, the expression of HIF-1a was significantly up-regulated in the necrotic area to label BMSCs, and co-cultured

BMSCs with xenogeneicantigen- extracted of the femoral head (Figure 6B), and the oxygen concentration was lower than $0.1 \%$, a hypoxic microenvironment was formed in the osteonecrotic area (Figure 6C). To evaluate the therapeutic effect of BMSC on early SONFH, subsequently, We used DiR cancellous bone (XACB) to construct tissue-engineered bone (XACB/BMSCs), and transplanted it to repair early SONFH (Figure 6L). At 2 days after operation, the fluorescence intensity of DiR was significantly decreased (Figure 6D and F), the expression of Bcl-2 was down-regulated, the expression of Bax and CASP3 was up-regulated (Figure $6 \mathrm{H}-\mathrm{K}$ ), and the proportion of TUNEL positive cells was more than $80 \%$ in the BMSCs transplantation group (Figure $6 \mathrm{E}$ and $\mathrm{G}$ ), which confirmed that a large number of BMSCs were apoptotic in the hypoxia environment of osteonecrotic area. At 12 weeks after operation, compared with that of the XACB group, there was no significant repair of the osteonecrotic area (Figure 6M-O) or formation of new bone tissue (Figure 6T-U) in the XACB/BMSC group, and there were no significant differences in the number of trabeculae (Figure 6P), trabecular thickness (Figure 6Q), new bone volume (Figure 6R), new bone volume fraction (Figure 6S) and osteogenic markers such as OPG, OCN, and Runx2 (Figure 6V-Y). These results indicate that a large number of transplanted BMSCs have hypoxia-induced apoptosis in the hypoxic microenvironment of osteonecrotic area, which seriously limits the effect of BMSCs transplantation.

Our in vitro studies have confirmed that Lnc Tmem235 inhibited hypoxia-induced apoptosis of BMSCs. So, we investigated whether LncTmem235mediated inhibition of hypoxia-induced apoptosis of BMSCs could improve the 
therapeutic effect of BMSCs on early SONFH. Lv-Lnc Tmem235 or Lv-Sh-Lnc Tmem235 were transfected into BMSCs to make BMSCs over-express or under-express Lnc Tmem235. Then, BMSCs were labeled with DiR, and co-cultured with $\mathrm{XACB}$ to construct tissue-engineered bone, which was transplanted to repair early SONFH model. At 2 days after operation, we evaluated whether Lnc Tmem235 could inhibit the apoptosis of transplanted BMSCs in the osteonecrotic area by the fluorescence intensity of DiR, the expression levels of GFP and BIRC5, and the proportion of TUNEL positive cells. The results showed that compared with that of the BMSCs/NC group, Lnc Tmem235 over-expression group had higher DiR fluorescence intensity (Figure 7B and D), GFP and BIRC5 expression levels (Figure $7 \mathrm{~A}$ ), and the proportion of TUNEL positive cells was significantly decreased (Figure 7C and E), while Lnc Tmem235 low-expression group had lower DiR fluorescence osteogenic markers (e.g., Runx2, OPN, OCN, and OPG) in the Lnc Tmem235 low-expression group were significantly lower than those of the BMSC/NC group $(P$ intensity (Figure 7B and D), GFP and BIRC5 expression levels (Figure 7A), and the proportion of TUNEL positive cells was increased (Figure 7C and E). These results suggest that Lnc Tmem235 inhibited apoptosis of BMSCs in the hypoxic microenvironment of osteonecrosis of the femoral head and promoted BMSC survival. At 12 weeks after operation, we evaluated the transplantation effect of BMSCs on early SONFH by micro-CT examination, HE staining, Masson staining and the level of osteogenic markers. The results showed that compared with BMSCs/NC group, the defect area in the Lnc Tmem235 over-expression group was completely repaired (Figure $7 \mathrm{G}$ ), the bone tissue tended to mature (Figure $7 \mathrm{~F}$ ), the number of trabeculae (Figure 7H), trabecular thickness (Figure 7I), new bone volume (Figure 7J), new bone volume fraction (Figure 7K), and the levels of osteogenic markers (e.g., Runx2, OPN, OCN, OPG) were significantly increased (Figure 7L-P). Conversely, there was no obvious repair in the defect area in the Lnc Tmem235 low-expression group (Figure $7 \mathrm{~F}-\mathrm{G}$ ), the number of trabecular (Figure 7H), trabecular thickness (Figure 7I), new bone volume (Figure $7 \mathrm{~J}$ ), new bone volume fraction (Figure $7 \mathrm{~K}$ ), and the levels of $<0.05$ ) (Figure 7L-P). These results suggest that Lnc Tmem235 improved the 
therapeutic effect of BMSCs on early SONFH by inhibiting hypoxia-induced apoptosis of BMSCs.

\section{Discussion}

BMSC transplantation has been shown to assist in repairing early SONFH. However, due to the hypoxic microenvironment in the necrotic area of the femoral head, transplanted BMSCs exhibit high rates of apoptosis, which seriously limits the osteogenic repair effect of BMSCs [35-36]. Therefore, methods that inhibit hypoxia-induced apoptosis of BMSCs are key to further improving the efficacy of BMSC transplantations. Recent studies have shown that LncRNAs regulate gene expression at many levels, such as at transcriptional and post-translational levels, and participate in various physiological activities [37]. In the present study, we elucidated a key role of Lnc Tmem235 in reducing hypoxia-induced apoptosis of BMSCs. Specifically, we found that Lnc Tmem235 can act as a ceRNA to competitively bind miR-34a-3p with BIRC5 mRNA to relieve the silencing effect of miR-34a-3p on BIRC5 mRNA, thus inhibiting hypoxia-induced apoptosis of BMSCs by promoting the expression of BIRC5. Additionally, we found that Lnc Tmem235 inhibition of hypoxia-induced apoptosis of BMSCs effectively improved the transplantation effect of BMSCs on treating early SONFH.

Hypoxia-induced apoptosis is one of the reasons for the limited efficacy of BMSC transplantations and it is a major obstacle to clinical applications [38]. Although improving mitochondrial function, clearing ROS, and interfering with apoptosis-related proteins can partially inhibit hypoxia-induced apoptosis of BMSCs, these effects remain unsatisfactory [15-18]. Therefore, it is important to develop new methods for improving the effects of BMSC transplantations. In recent years, with the continuous development of sequencing technology, the biological functions of LncRNAs have been continuously explored, and studies have shown that LncRNAs play an important role in regulating apoptosis. For example, Lnc ZFAS1 can inhibit apoptosis induced by calcium overload [39]. Moreover, LncRNAs are also involved in the regulation of stress responses, such as hypoxia. For example, Lnc P21 can form a 
positive-feedback loop with HIF-1a, promoting hypoxia glycolysis in tumor cells [40]. However, the regulatory role of LncRNA in the process of hypoxia-induced apoptosis

328 has remained poorly understood. In the present study, we screened out hypoxia-reactive Lnc Tmem235 in a BMSC hypoxic model by using LncRNA microarrays. Under hypoxic conditions, the expression of Lnc Tmem235 was down-regulated, and its expression continued to be down-regulated with the aggravation of hypoxia and an increased apoptotic rate, suggesting that Lnc Tmem235 may be related to hypoxia-induced apoptosis of BMSCs. Subsequently, we conducted functional experiments on Lnc Tmem235, and the results confirmed that Lnc Tmem235 could inhibit the hypoxia-induced apoptosis of BMSCs. These results indicate that LncRNAs regulate hypoxia-induced apoptosis, which provide novel targets for inhibiting hypoxia-induced apoptosis of BMSCs.

LncRNAs participate in various physiological activities mainly by regulating gene expression [41]. In our present study, we investigated the mechanism by which Lnc Tmem235 inhibited hypoxia-induced apoptosis of BMSCs. Under hypoxia, we analyzed the gene expression profile of BMSCs-Lnc Tmem235 by microarray, and found that the expression of BIRC5 was significantly up-regulated. Then, we verified the regulatory relationship between Lnc Tmem235 and BIRC5 in a BMSC hypoxic model. Specifically, we found that LncTmem235 promoted the expression of BIRC5. BIRC5 is a member of the apoptosis-inhibitor family, which can block the process of apoptosis by inhibiting Caspase activity [30-33]. To study the role of BIRC5 in inhibiting hypoxia-induced apoptosis of BMSCs by Lnc Tmem235, we knocked out BIRC5 while up-regulating Lnc Tmem235, which completely blocked the anti-apoptotic effect of Lnc Tmem235. Alternatively, we also up-regulated BIRC5 while down-regulating Lnc Tmem235, which mitigated hypoxia-induced apoptosis of BMSCs. These results indicated that Lnc Tmem235 inhibited hypoxia-induced apoptosis of BMSCs by regulating the expression of BIRC5.

LncRNA can regulate gene expression through a variety of mechanisms, including pre-transcriptional regulation (e.g., histone modification and DNA methylation), transcriptional regulation (e.g., enhancer activity, transcriptional 
interference, and control of transcription factors), and post-transcriptional regulation (e.g., regulation of alternative splicing, regulation of RNA subcellular location, regulation of RNA stability) [42-44]. It is noteworthy that more and more evidence has shown that LncRNA can be used as a ceRNA to regulate the expression of target genes through miRNAs. For example, Lnc RoR can be used as a ceRNA to regulate the expression of Oct4, Nanog, and Sox2, which then interferes with the self-renewal of human embryonic stem cells [45]. Lnc SPAG5-AS1 can be used as a ceRNA to regulate the expression of SPAG5 to inhibit autophagy and apoptosis through the AKT/mTOR pathway [46]. In our present study, our bioinformatic predictions showed that Lnc Tmem235 and BIRC5 mRNA have the same binding site on miR-34a-3p, and that Lnc Tmem235 and BIRC5 mRNA satisfy conditions for competitive binding to miR-34a-3p. Subsequently, our results confirmed that Lnc Tmem235 could be used as a ceRNA to bind miR-34a-3p competitively with BIRC5 mRNA to relieve the silencing effect of miR-34a-3p on BIRC5 mRNA, and thereby regulate the expression of BIRC5. Collectively, these findings reveal a mechanism of Lnc Tmem235 as a ceRNA regulating gene expression from the level of post-transcriptional regulation.

In our present study, we used BMSCs to repair an early SONFH model, but the transplantation effect was not satisfactory. The main reason for this result was that a large number of BMSCs were apoptotic in the hypoxic microenvironment in the femoral head necrotic area, which seriously limited the transplantation effect of BMSCs on early SONFH. Strikingly, our results in vitro confirmed that Lnc Tmem235 inhibited hypoxia-induced apoptosis of BMSCs. Furthermore, to evaluate the effect of Lnc Tmem235 in vivo, we transplanted BMSCs with an overexpression Lnc-Tmem235 construct to repair our early SONFH model. The results showed that Lnc-Tmem235-mediated inhibition of hypoxia-induced apoptosis of BMSCs effectively improved the therapeutic efficacy of BMSC transplantation.

In summary, we elucidated that Lnc Tmem235 inhibited hypoxia-induced apoptosis in BMSCs by regulating miR-34a-3p/BIRC5, and that Lnc-Tmem235mediated inhibition of hypoxia-induced apoptosis in BMSCs effectively improved the transplantation effect of BMSCs on early SONFH (Figure 8). Collectively, our 
findings provide a new target and method for reducing hypoxia-induced apoptosis of BMSCs and improving the efficacy of BMSC transplantations.

\section{Methods}

\section{Animals}

We extracted BMSCs from young male Sprague-Dawley (SD) rats (20-30 g) and built SONFH models using adult male SD rats (500-600 g). SD rats were provided by the Laboratory Animal Center of Guizhou Medical University (Guiyang, China). All experiments were approved by the Experimental Animal Ethics Committee of Guizhou Medical University (NO.1900677), and the experimental facility certificate number is SYXK (Qian) 2018-0001. All procedures were performed in accordance with our Institutional Guidelines for Animal Research, and the investigation conformed to the Guide for the Care and Use of Laboratory Animals published by the US National Institutes of Health (NIH Publication No. 85-23, revised in 1996).

\section{BMSC cultures}

The bilateral femurs and tibia of male SD rats (weighing 20-30 g) were removed, and the bone marrow fluid was obtained by flushing the bone marrow cavity under aseptic conditions. The bone marrow fluid was diluted with phosphate-buffered saline (PBS; Hyclon, USA) at a ratio of 1:1 and was then transferred into a single cell suspension, after which it was centrifuged at $600 \mathrm{~g} / \mathrm{min}$ for $5 \mathrm{~min}$. The precipitated bone marrow was slowly added to the Percoll separation solution $(1.073 \mathrm{~g} / \mathrm{mL}$; Pharmacia, USA) along the tube wall, centrifuged at $900 \mathrm{~g} / \mathrm{min}$ for $30 \mathrm{~min}$, and nucleated cells were absorbed. We then washed the cells with PBS, centrifuged at 600 $\mathrm{g} / \mathrm{min}$ for $15 \mathrm{~min}$. The cells were resuspended with complete L-glutamine Dulbecco's Modified Eagle Medium (L-DMEM; Gibco, USA) containing 10\% fetal bovine serum (FBS; Gibco, USA) and 1\% double antibody (Hyclon, USA), after which they were cultured at $37^{\circ} \mathrm{C}$ and $5 \% \mathrm{CO}_{2}$. When the primary BMSC confluence reached about $90 \%$ of the bottom of the culture bottle, the cells were digested with $0.25 \%$ trypsin $0.02 \%$ ethylenediaminetetraacetic acid (EDTA; Gibco, USA) at $37^{\circ} \mathrm{C}$, and the cells were passaged at a 1:3 ratio. Second-generation BMSCs were used for subsequent 
experiments.

\section{Osteogenic differentiation of BMSCs}

Second-generation BMSCs were inoculated in six-well plates. When the cell confluence was about $60 \%$ at the bottom of the culture bottle, according to the instructions of the BMSC osteogenesis induction kit (Cyagen Biosciences, USA), the experimental group was replaced with BMSC osteogenesis induction medium, while the control group was remained in complete L-DMEM medium. After two weeks of osteogenesis induction, the cells were fixed, alkaline phosphatase (ALP) (Cyagen Biosciences, USA) staining was used to detect ALP activity, and 0.1\% alizarin red (Cyagen Biosciences, USA) staining was used to identify calcium nodules.

\section{Lipogenic differentiation of BMSCs}

Second-generation BMSCs were inoculated in six-well plates. When the cell fusion degree reached $100 \%$ or reached over-fusion, according to the description of the BMSC adipogenic induction kit (Cyagen Biosciences, USA), the experimental group was replaced with BMSC adipogenic differentiation medium, while the control group was remained in complete L-DMEM medium. After three weeks of adipogenic induction, lipid droplets were identified by oil-red-O staining (Cyagen Biosciences, USA).

\section{Chondrogenic differentiation of BMSCs}

Second-generation BMSCs were inoculated in six-well plates. When the cell fusion degree reached about $60 \%$, according to the description of the BMSC chondrogenic induction kit (Cyagen Biosciences, USA), the experimental group was replaced with BMSC chondrogenic induction medium, while the control group remained in complete L-DMEM medium. After four weeks of chondrogenic induction, acidic mucopolysaccharides in cartilage tissue were identified by alixin-blue staining (Cyagen Biosciences, USA).

\section{Identification of surface antigens of BMSCs}

The density of second-generation BMSCs was adjusted to $2 \times 10^{7}$ cells $/ \mathrm{mL}$. The control group received $50 \mathrm{uL}$ of buffer while the single-label group received $5 \mathrm{uL}$ of hamster anti-CD29-AF647 (BD, USA), mouse anti-CD90-PECyTM7 (BD, USA), 
mouse anti-CD106-PE (BD, USA), mouse anti-CD11b-V450 (BD, USA), or mouse anti-CD45-FITC (BD, USA) to each branch-flow sampling tube, after which $45 \mathrm{uL}$ of buffer was added to each tube. The multicolor group received $5 \mathrm{uL}$ of each antibody into a one-branch-flow sampling tube, and then add $25 \mathrm{uL}$ of buffer was added. Next, $50 \mathrm{uL}$ of cell suspension was added to each flow tube, the tubes were incubated at room temperature for $30 \mathrm{~min}$, washed twice with staining buffer, and then $500 \mathrm{uL}$ of buffer was added to each tube for detection by flow cytometry (Beckman, USA).

\section{Cell hypoxia model}

To induce hypoxia, second-generation BMSCs were continuously treated with mixed gas at an oxygen concentration of $0 \%$, nitrogen concentration of $95 \%$, and carbon dioxide concentration of $5 \%$ for $48 \mathrm{~h}$.

\section{ATP content}

Second-generation BMSCs were collected into centrifuge tubes. According to the instructions of the ATP content detection kit (Solarbio, China), $1 \mathrm{~mL}$ of the extracted liquid was added to five million cells, which were crushed by ultrasound for $1 \mathrm{~min}$ (ice bath, $200 \mathrm{~W}$, ultrasonic time was $2 \mathrm{~s}$, stopping time was $1 \mathrm{~s}$ ) and centrifuged at $10000 \mathrm{~g} / \mathrm{min}$ for $10 \mathrm{~min}$. Then, the supernatant of each sample was collected into an Eppendorf tube, and was added to $500 \mu \mathrm{L}$ of chloroform prior to centrifugation at $10000 \mathrm{~g} / \mathrm{min}$ for $3 \mathrm{~min}$. The supernatant was fully mixed with the working solution and, after $10 \mathrm{sec}$, the absorbance value A1 under $340 \mathrm{~nm}$ was determined via an enzyme meter (Biotech, USA). Then a 96-well plate was placed into an incubator at $37^{\circ} \mathrm{C}$ for $180 \mathrm{~s}$, and the absorbance value A2 at $190 \mathrm{~s}$ was determined. The absorbance values of the sample solution and ATP standard solution were determined at the same time, and finally the ATP content was calculated as follows: ATP content ( $\mu \mathrm{mol} / 10^{6}$ cells $)=0.125 \times \Delta \mathrm{A}$ sample $\div \Delta \mathrm{A}$ standard, $\Delta \mathrm{A}$ sample $=\mathrm{A} 2$ sample $-\mathrm{A} 1$ sample, $\Delta \mathrm{A}$ standard $=\mathrm{A} 2$ standard $-\mathrm{A} 1$ standard.

\section{Mitochondrial membrane potential}

After hypoxia treatment to second-generation BMSCs, the cells were washed with PBS, and the reaction mixture was prepared according to the instructions of the mitochondrial membrane potential detection kit (KeyGEN BioTECH, China). The 
reaction mixture was added, incubated at $37^{\circ} \mathrm{C}$ for $30 \mathrm{~min}$, washed three times with PBS, and the fluorescence was observed under a laser confocal microscope (Zeiss, Germany).

\section{ROS content}

After hypoxia treatment of second-generation BMSCs, the cells were washed with PBS buffer and stained according to the instructions of the DCFH-DA fluorescence probe kit (Sigma, Germany). The cells were incubated in a $5 \% \mathrm{CO}_{2}$ incubator at $37^{\circ} \mathrm{C}$ for $30 \mathrm{~min}$, and green fluorescence was observed by laser confocal microscopy.

\section{TUNNEL/DAPI}

Second-generation BMSCs were fixed with 4\% paraformaldehyde (Solarbio, China) at room temperature for $30 \mathrm{~min}$ and permeabilized with $0.3 \%$ Triton $\mathrm{X}-100$ (Solarbio, China) for $6 \mathrm{~min}$. Then, TUNEL detection solution (Beytime, China) was added and reacted at $37^{\circ} \mathrm{C}$ for $60 \mathrm{~min}$ in the dark, after which the samples were washed with PBS and stained with DAPI (Solarbio, China) for $5 \mathrm{~min}$.

\section{Annexin V-FITC/PI}

Second-generation BMSCs were washed with PBS, and then $5 \mu \mathrm{L}$ Annexin V-FITC and $5 \mu \mathrm{L}$ PI were directly added according to the instructions of the Annexin V-FITC apoptosis detection kit (BD, USA). The cells were gently vortexed and incubated at room temperature in the dark for $15 \mathrm{~min}$, and were then detected by flow cytometry.

\section{Lentiviral transfections}

Lentiviruses were purchased from China Shanghai Genechem Co., Ltd., according to the best $\mathrm{MOI}(\mathrm{MOI}=80)$ and the best transfection condition (Eni.s+Polybrane) found during our transfection pre-experiments. Second-generation BMSCs were infected with these lentiviruses, and a blank control and negative control were also established at this time. After $12 \mathrm{~h}$, the culture medium was changed to complete L-DMEM. On the fourth day after infection, the stable strain was screened by adding $2 \mu \mathrm{g} / \mathrm{mL}$ of puromycin. When all cells in the blank control died, the concentration of puromycin was reduced to $1 \mu \mathrm{g} / \mathrm{mL}$ to maintain the screening. 


\section{Microarray and bioinformatic analyses}

The total RNA of three pairs of cell samples treated with regular oxygen and hypoxia were extracted by Trizol reagent (Invitrogen, USA). The RNA quality was confirmed by formaldehyde agarose gel electrophoresis and quantified by a NanoDrop ND-1000. Double-stranded cDNA was synthesized from total RNA samples without rRNA and was then labeled with cDNA and hybridized to SD rat LncRNA expression microarray v3.0 $(8 \times 60 \mathrm{~K}$, Arraystar, USA). After cleaning the microarray, the slides were scanned with an Agilent Microarray Scanner (Agilent p/n G2565BA). Raw data were extracted as paired files using the Agilent Feature Extraction. The differentially-expressed genes were identified by a random variance model. Paired t-tests were used to calculate P values. The thresholds of up-regulated and down-regulated genes are expressed as fold-change $(\mathrm{FC})>2.0$ and $\mathrm{P}<0.05$, respectively. Hierarchical clustering was performed using clustering software to analyze the expression patterns of LncRNAs and mRNAs. The gene co-expression Network between LncRNAs and mRNAs was analyzed by Cytoscape software. GO and KEGG analysis were performed to cluster the differentially-expressed mRNAs $($ FC $>2)$. The microarray data were then stored in the NCBI gene expression database.

\section{Double-luciferase reporter assays}

After BMSCs were transfected with lentiviruses containing the luciferase reporter gene, BMSCs were washed with PBS, lysed with cell lysate according to the instructions of the luciferase assay kit (Solarbio, China), and then the sea-renal luciferase reaction substrate was added. Finally, luciferase activity was detected at a wavelength of $465 \mathrm{~nm}$ by a multifunction enzyme marker (Biotech, USA).

\section{RIP}

RIP was performed using a Magna RNA-binding protein immunoprecipitation kit (Millipore, USA) according to the manufacturer's instructions. First, Ago2 antibody-magnetic beads were incubated with cell lysates, and IgG negative controls were set up at the same time. Then, immuno-coprecipitation was conducted to isolate all RNA-proteins bound to Ago2. Finally, purified RNAs were extracted and 
determined by real-time PCR to confirm the presence of the binding targets.

\section{Real-time quantitative PCR}

We extracted RNA from BMSCs via column affinity purification (QIAGEN, Germany) and synthesized complementary DNAs (cDNAs) using M-MuLV RT Master Mix with Oligo(dT) (Sangon Biotech, China). We performed real-time PCR on a StepOnePlus system (Applied Biosystems, USA) in 96-well plates with specific primers and SYBR Green Mix (Sangon Biotech). Rabbit primers (Sangon Biotech) were as follows: Lnc-Tmem235-F: GGGAGAAGGTCATCTCAGGCA; Lnc-Tmem235-R: GCTGTTGCTGCCTTTCTCAAGT; Lnc-LOC102553514-F: CAG CGTCAGACCTCCGTCTA; Lnc-LOC102553514-R: TTAAGCATTGCGGGTGCCA A; BIRC5-F: TGCCTTACGCTGAGCCTTTGC; BIRC5-R: GCCTGGAAAGCTGG GACAAGTG; miRNA-34a-3p-F: CGCGCGAATCAGCAAGTATACT; miRNA-34a3p-R: AGTGCAGGGTCCGAGGTATT; miRNA-34a-3p-RT: GTCGTATCCAGTGC AGGGTCCGAGGTATTCGCACTGGATACGACTAGGGC; ACTB-F: CACCCGCG AGTACAACCTTC; and ACTB-R: CCCATACCCACCATCACACC. We calculated the fold-change in RNA expression compared to that of the control using the $\Delta \Delta \mathrm{Ct}$ method.

\section{Western blotting}

The cells were lysed with RIPA cell lysate (Beytime, China) and centrifuged at $13000 \mathrm{~g} / \mathrm{min}$ for $10 \mathrm{~min}$. The supernatant was obtained and the protein was quantified by a BCA protein concentration detection kit (Solarbio, Beijing, China). SDS-PAGE gels were prepared, the same amount of protein was added for electrophoresis, and then the protein was transferred to a PVDF membrane. Rabbit anti-BIRC5 (1:3000; ab469; Abcam, UK), rabbit anti-Bcl-2 (1:500; bs-0032R; Bioss, China), rabbit anti-Caspase-3 (1:500; ab4051; Abcam, UK), rabbit anti-Bax (1:1500; bs-28034R; Bioss), mouse anti- $\beta$-actin (1:2500; ab8226; Abcam), Rabbit anti-CASP-3/Cleaved-CASP-3 (1:500; ab13847; Abcam), Rabbit anti-CASP-9/Cleaved-CASP-9 (1:1000; ab25758; Abcam), Rabbit anti-PARP1/Cleaved-PARP1 (1:10000; ab191217; Abcam), Rabbit anti-Argonaute 2 (1:800; ab5072; Abcam), mouse anti-OCN (10 $\mu \mathrm{g} / \mathrm{mL}$; ab13420; Abcam, UK), rabbit 
anti-OPG (1:100; bs-20624R; Bioss, China), rabbit anti-Runx2 (1:200; AV36678; Sigma-Aldrich, Germany), and rabbit anti-OPN (1:200; ab8448; Abcam) were used for primary-antibody reactions. HRP-conjugated mouse anti-rabbit IgG was used for secondary-antibody reactions. ECL (Merck Millipore) photoluminescence solution was used for exposure. Finally, the images were collected by a gel imaging system (Clinx Science Instruments, Ltd., China) and quantitatively analyzed by ImageJ software (1.4.3.67).

\section{XACB}

We removed bone cortex and cartilage from fresh porcine vertebrae, and the cancellous bone was made into a cylinder with a diameter of $5 \mathrm{~mm}$ and a height of 10 $\mathrm{mm}$. The bone was washed repeatedly in an ultrasonic cleaning machine. Then, samples were deproteinized with 30\% hydrogen peroxide (Chengdu Jinshan Chemical Reagent CO., LTD., China) for $48 \mathrm{~h}$ while stirring intermittently, and by replacing hydrogen peroxide every $24 \mathrm{~h}$; Samples were then degreased with 1:1 chloroform/methanol (Chengdu Jinshan Chemical Reagent CO., LTD., China) for 24 $\mathrm{h}$ while stirring intermittently, and 1:1 chloroform/methanol was changed every $12 \mathrm{~h}$; Samples were freeze-dried at $-50^{\circ} \mathrm{C}$ for $24 \mathrm{~h}$, and were sterilized with ethylene oxide for $24 \mathrm{~h}$, followed by being aseptically packaged until further use.

\section{Early SONFH model}

A total of 150 adult male SD rats were intravenously injected twice with lipopolysaccharides (10 $\mu \mathrm{g} / \mathrm{kg}$; Sigma, Germany) once a day. After the second injection of lipopolysaccharides, methylprednisolone (40 mg/kg; Pfizer, USA) was injected three times into the gluteal muscle immediately, once a day, and was weighed before each injection.

\section{Biocompatibility of XACB with BMSCs}

The XACB was made into a small cylinder with a diameter of $5 \mathrm{~mm}$ and a height of $5 \mathrm{~mm}$, after which it was soaked in complete L-DMEM medium for $24 \mathrm{~h}$. A BMSC suspension $\left(1 \times 10^{7}\right.$ cells $\left./ \mathrm{mL}\right)$ was dropped into XACB, incubated in an incubator at $37^{\circ} \mathrm{C}$ and $5 \% \mathrm{CO}_{2}$ for $3 \mathrm{~h}$, and then complete L-DMEM medium was slowly added along the wall of the culture plate to just pass XACB, after which the culture was 
continued in the incubator. On the sixth day, the growth of BMSCs on the surface of XACB was observed by scanning electron microscopy (HITACHI, Japan).

\section{Tissue-engineered bone}

After Lv-Lnc Tmem235 and Lv-Sh-Lnc Tmem235 were transfected into BMSCs, BMSCs were dropped into XACB and co-cultured according to the above steps to construct tissue-engineered bone.

\section{Tissue-engineered bone transplantation}

The skin of the right hip of SD rats was cleaned one week before operation. Penicillin (50000 U/Kg; CSPC, China) was injected intramuscularly at $1 \mathrm{~h}$ before operation to prevent infection. Then, 3\% pentobarbital sodium $(1 \mathrm{~mL} / \mathrm{kg}$; Merck, Germany) was injected intravenously to anesthetize rats. Rats were then placed in a prone position, and the operated area was disinfected. A posterolateral straight incision of the hip joint was made to expose the hip joint, and then the femoral head was rotated externally. At the junction of bone and cartilage, a sterile spherical drill with a diameter of $2 \mathrm{~mm}$ was used to grind this junction from the posterolateral to the anterior medial side, and the depth was about $3 \mathrm{~mm}$. The necrotic bone tissue was completely scraped, and the tissue-engineered bone was implanted according to the experimental group, after which the pore was filled with a gelatin sponge (Alicon, China). Penicillin was used for $3 \mathrm{~d}$ after the operation to prevent infection.

\section{Live imaging of animals}

The BMSCs were labeled with DiR (YEASEN, Shanghai, China) fluorescent dyes before the operation, 48 hours after BMSCs transplantation, the fluorescence intensity of the femoral head necrosis area was detected by a small animal imaging system, chloral hydrate $(10 \%, 3 \mathrm{~mL} / \mathrm{Kg})$ was anesthetized in the abdominal cavity, and the rats were placed for imaging platform, take fluorescence pictures and calculate fluorescence intensity.

\section{micro-CT}

At 4, 8, and 12 weeks after BMSCs transplantation, femoral head tissue was scanned using the Bruker micro-CT system (SkyScan1276, Germany) under uniform conditions (voltage $85 \mathrm{kV}$, current $200 \mu \mathrm{A}$, resolution $6.5 \mu \mathrm{m}$ ). N-Recon software was 
used for 3D image reconstruction, and CT-AN software was used for 3D analysis. At the same time, the number of trabeculae, trabecular thickness, new bone volume and volume fraction were detected.

\section{TUNEL staining}

The femoral head was fixed overnight in $4 \%$ formaldehyde PBS solution, and was then embedded in paraffin wax and made into $3-\mu$ m-thick sections. According to the instructions of the TUNEL detection kit, the sections were stained and the positive cell rate was statistically analyzed.

\section{H\&E and Masson staining}

Twelve weeks after operation, the bone tissue was decalcified, dehydrated, and embedded in paraffin to make 3 - $\mu$ m-thick sections, which were stained according to the HE staining kit (Solarbio, China). Paraffin sections were dewaxed to water, stained according to the Masson staining kit (Solarbio, China), and were then made transparent, sealed, and examined under a microscope.

\section{Immunofluorescence}

At 12 weeks after operation, the tissue of the femoral head was dehydrated, made transparent, waxed, and embedded, and was then made into $3-\mu \mathrm{m}$-thick sections. Then, the tissue was dewaxed, and rabbit anti-BIRC5 (1/100; ab134170; Abcam, UK) and rabbit anti-GFP (1/1000; ab6556; Abcam, UK) were used for primary-antibody reactions. Goat Anti-Rabbit IgG (Alexa Fluor ${ }^{\circledR}$ 488, 1/800, ab150077, Abcam, UK) and Goat Anti-Rabbit IgG (Alexa Fluor® 594, 1/800, ab150080, Abcam, UK) were used for secondary-antibody reactions. DAPI re-staining of the nucleus was performed, and the sections were sealed with a sealing solution containing an anti-fluorescent quenching agent, after which images were observed and collected under a fluorescent microscope.

\section{Statistical analysis}

All statistical data were calculated and graphed using GraphPad Prism software version 6 (GraphPad Software, San Diego, California, USA). To assess statistical significance, we used a Kolmogorov-Smirnov test to analyze the normality of the data. Two-tailed unpaired Student's $t$ tests were used for analyses involving only two 
groups for comparison, and one-way ANOVA with Tukey's post-hoc test were used for analyses involving more than two groups. All data are expressed as means \pm standard deviations (SDs). $P<0.05$ was considered statistically significant.

\section{Data availability}

Additional data or reagents are available from the corresponding author upon reasonable request.

\section{Conflict of interest}

The authors declare that they have no conflict of interest.

\section{References}

[1] Zhang Q, Lv J, Jin L. Role of coagulopathy in glucocorticoid-induced osteonecrosis of the femoral head. J Int Med Res, 2018, 46(6): 2141-2148.

[2] Li T, Zhang Y, Wang R, Xue Z, Li S, Cao Y, Liu D, Niu Y, Mao X, Wang X, Li W, Guo Q, Guo M, Lin N, Chen W. Discovery and validation an eight-biomarker serum gene signature for the diagnosis of steroid-induced osteonecrosis of the femoral head. Bone, 2019, 122: 199-208.

[3] Fang S, Li Y, Chen P. Osteogenic effect of bone marrow mesenchymal stem cell-derived exosomes on steroid-induced osteonecrosis of the femoral head. Drug Des Devel Ther, 2018, 13: 45-55.

[4] Maruyama M, Nabeshima A, Pan CC, Behn AW, Thio T, Lin T, Pajarinen J, Kawai T, Takagi M, Goodman SB, Yang YP. The effects of a functionally-graded scaffold and bone marrow-derived mononuclear cells on steroid-induced femoral head osteonecrosis. Biomaterials, 2018, 187: 39-46.

[5] Hernigou P, Flouzat-Lachaniette CH, Delambre J, Poignard A, Allain J, Chevallier

N, Rouard H. Osteonecrosis repair with bone marrow cell therapies: state of the clinical art. Bone, 2015, 70: 102-109.

[6] Xing M, Wang X, Wang E, Gao L, Chang J. Bone tissue engineering strategy based on the synergistic effects of silicon and strontium ions. Acta Biomater, 2018, 72: 381-395.

[7] Weinstein RS, Hogan EA, Borrelli MJ, Liachenko S, O'Brien CA, Manolagas SC. The Pathophysiological Sequence of Glucocorticoid-Induced Osteonecrosis of the 
Femoral Head in Male Mice. Endocrinology, 2017, 158(11): 3817-3831.

[8] Li Z, Jiang C, Li X, Wu WKK, Chen X, Zhu S, Ye C, Chan MTV, Qian W. Circulating microRNA signature of steroid-induced osteonecrosis of the femoral head. Cell Prolif, 2018, 51(1): e12418.

[9] Ciapetti G, Granchi D, Fotia C, Savarino L, Dallari D, Del Piccolo N, Donati DM, Baldini N. Effects of hypoxia on osteogenic differentiation of mesenchymal stromal cells used as a cell therapy for avascular necrosis of the femoral head. Cytotherapy, 2016, 18(9): 1087-1099.

[10] Kao GS, Tu YK, Sung PH, Wang FS, Lu YD, Wu CT, Lin RLC, Yip HK, Lee MS. MicroRNA-mediated interacting circuits predict hypoxia and inhibited osteogenesis of stem cells, and dysregulated angiogenesis are involved in osteonecrosis of the femoral head. Int Orthop, 2018, 42(7): 1605-1614.

[11] Mangi AA, Noiseux N, Kong D, He H, Rezvani M, Ingwall JS, Dzau VJ. Mesenchymal stem cells modified with Akt prevent remodeling and restore performance of infarcted hearts. Nat Med, 2003, 9(9): 1195-1201.

[12] Balsam LB, Wagers AJ, Christensen JL, Kofidis T, Weissman IL, Robbins RC. Haematopoietic stem cells adopt mature haematopoietic fates in ischaemic myocardium. Nature, 2004, 428(6983): 668-673.

[13] Lien CF, Lee WS, Wang IC, Chen TI, Chen TL, Yang KT. Intermittent hypoxia-generated ROS contributes to intracellular zinc regulation that limits ischemia/reperfusion injury in adult rat cardiomyocyte. J Mol Cell Cardiol, 2018, 118 : 122-132.

[14] Greijer AE, van der Wall E. The role of hypoxia inducible factor 1 (HIF-1) in hypoxia induced apoptosis. J Clin Pathol, 2004, 57(10): 1009-1014.

[15] Baldari S, Di Rocco G, Piccoli M, Pozzobon M, Muraca M, Toietta G. Challenges and Strategies for Improving the Regenerative Effects of Mesenchymal Stromal Cell-Based Therapies. Int J Mol Sci, 2017, 18(10): 2087.

[16] Liu XB, Wang JA, Ji XY, Yu SP, Wei L. Preconditioning of bone marrow mesenchymal stem cells by prolyl hydroxylase inhibition enhances cell survival and angiogenesis in vitro and after transplantation into the ischemic heart of rats. Stem 
Cell Res Ther, 2014, 5(5): 111.

[17] Gulshan M, Yaku K, Okabe K, Mahmood A, Sasaki T, Yamamoto M, Hikosaka

K, Usui I, Kitamura T, Tobe K, Nakagawa T. Overexpression of Nmnat3 efficiently increases NAD and NGD levels and ameliorates age-associated insulin resistance. Aging Cell, 2018, 17(4): e12798.

[18] Hershberger KA, Martin AS, Hirschey MD. Role of $\mathrm{NAD}^{+}$and mitochondrial sirtuins in cardiac and renal diseases. Nat Rev Nephrol, 2017, 13(4): 213-225.

[19] Jiao L, Li M, Shao Y, Zhang Y, Gong M, Yang X, Wang Y, Tan Z, Sun L, Xuan L, Yu Q, Li Y, Gao Y, Liu H, Xu H, Li X, Zhang Y, Zhang Y. IncRNA-ZFAS1 induces mitochondria-mediated apoptosis by causing cytosolic $\mathrm{Ca}^{2+}$ overload in myocardial infarction mice model. Cell Death Dis, 2019, 10(12): 942.

[20] Zhang K, Zhang M, Yao Q, Han X, Zhao Y, Zheng L, Li G, Liu Q, Chang Y, Zhang P, Cui H, Shi Z, Chen T, Yao Z, Han T, Hong W. The hepatocyte-specifically expressed lnc-HSER alleviates hepatic fibrosis by inhibiting hepatocyte apoptosis and epithelial-mesenchymal transition. Theranostics, 2019, 9(25): 7566-7582.

[21] Rossi MN, Antonangeli F. LncRNAs: New Players in Apoptosis Control [J]. Int J Cell Biol, 2014, 2014: 473857.

[22] Li K, Zhong S, Luo Y, Zou D, Li M, Li Y, Lu Y, Miao S, Wang L, Song W. A long noncoding RNA binding to QKI-5 regulates germ cell apoptosis via p38 MAPK signaling pathway. Cell Death Dis, 2019, 10(10): 699.

[23] Yang LX, Wu J, Guo ML, Zhang Y, Ma SG. Suppression of long non-coding RNA TNRC6C-AS1 protects against thyroid carcinoma through DNA demethylation of STK4 via the Hippo signalling pathway. Cell Prolif, 2019, 52(3):e12564.

[24] Wang Y, He L, Du Y, Zhu P, Huang G, Luo J, Yan X, Ye B, Li C, Xia P, Zhang G, Tian Y, Chen R, Fan Z. The long noncoding RNA lncTCF7 promotes self-renewal of human liver cancer stem cells through activation of Wnt signaling. Cell Stem Cell, $2015,16(4): 413-25$.

[25] Li DY, Busch A, Jin H, Chernogubova E, Pelisek J, Karlsson J, Sennblad B, Liu S, Lao S, Hofmann P, Bäcklund A, Eken SM, Roy J, Eriksson P, Dacken B, Ramanujam D, Dueck A, Engelhardt S, Boon RA, Eckstein HH, Spin JM, Tsao PS, 
Maegdefessel L. H19 Induces Abdominal Aortic Aneurysm Development and Progression. Circulation, 2018, 138(15): 1551-1568.

[26] Wang Y, Zhang L, Zheng X, Zhong W, Tian X, Yin B, Tian K, Zhang W. Long non-coding RNA LINC00161 sensitises osteosarcoma cells to cisplatin-induced apoptosis by regulating the miR-645-IFIT2 axis. Cancer Lett, 2016, 382(2): 137-146. [27] Kong X, Liu CX, Wang GD, Yang H, Yao XM, Hua Q, Li XY, Zhang HM, Ma MZ, Su Q, Lv K. LncRNA LEGLTBC Functions as a ceRNA to Antagonize the Effects of miR-34a on the Downregulation of SIRT1 in Glucolipotoxicity-Induced INS-1 Beta Cell Oxidative Stress and Apoptosis. Oxid Med Cell Longev, 2019, 2019: 4010764.

[28] Wang K, Long B, Zhou LY, Liu F, Zhou QY, Liu CY, Fan YY, Li PF. CARL lncRNA inhibits anoxia-induced mitochondrial fission and apoptosis in cardiomyocytes by impairing miR-539-dependent PHB2 downregulation. Nat Commun, 2014, 5:3596.

[29] Yang X, Xing G, Liu S, Li B, He Y, Wang F. LncRNA LOXL1-AS1 promotes endometrial cancer progression by sponging miR-28-5p to upregulate RAP1B expression. Biomed Pharmacother, 2020, 125:109839.

[30] Wheatley SP, Altieri DC. Survivin at a glance. J Cell Sci, 2019, 132(7): jes223826.

[31] Wang R, Kang Y, Löhr CV, Fischer KA, Bradford CS, Johnson G, Dashwood WM, Williams DE, Ho E, Dashwood RH. Reciprocal regulation of BMF and BIRC5 (Survivin) linked to Eomes overexpression in colorectal cancer. Cancer Lett, 2016, 381(2): 341-348.

[32] Rathore R, McCallum JE, Varghese E, Florea AM, Büsselberg D. Overcoming chemotherapy drug resistance by targeting inhibitors of apoptosis proteins (IAPs). Apoptosis, 2017, 22(7): 898-919.

[33] Santarelli A, Mascitti M, Lo Russo L, Sartini D, Troiano G, Emanuelli M, Lo Muzio L. Survivin-Based Treatment Strategies for Squamous Cell Carcinoma. Int J Mol Sci, 2018, 19(4): 971.

[34] Tay Y, Rinn J, Pandolfi PP. The multilayered complexity of ceRNA crosstalk and 
competition. Nature, 2014, 505(7483):344-52.

[35] Fan L, Zhang C, Yu Z, Shi Z, Dang X, Wang K. Transplantation of hypoxia preconditioned bone marrow mesenchymal stem cells enhances angiogenesis and osteogenesis in rabbit femoral head osteonecrosis. Bone, 2015, 81: 544-553.

[36] Yang QH, Li GY, Gao YS, Zhang CQ. Discrepant hypoxia tolerance aggravates subchondral delamination in osteonecrosis of the femoral head. Int Orthop, 2019, 43(3): 753-754.

[37] Kopp F, Mendell JT. Functional Classification and Experimental Dissection of Long Noncoding RNAs. Cell, 2018, 172(3):393-407.

[38] Lin M, Liu X, Zheng H, Huang X, Wu Y, Huang A, Zhu H, Hu Y, Mai W, Huang Y. IGF-1 enhances BMSC viability, migration, and anti-apoptosis in myocardial infarction via secreted frizzled-related protein 2 pathway. Stem Cell Res Ther, 2020, 11(1):22.

[39] Jiao L, Li M, Shao Y, Zhang Y, Gong M, Yang X, Wang Y, Tan Z, Sun L, Xuan L, Yu Q, Li Y, Gao Y, Liu H, Xu H, Li X, Zhang Y, Zhang Y. lncRNA-ZFAS1 induces mitochondria-mediated apoptosis by causing cytosolic $\mathrm{Ca}^{2+}$ overload in myocardial infarction mice model. Cell Death Dis, 2019, 10(12):942.

[40] Yang F, Zhang H, Mei Y, Wu M. Reciprocal regulation of HIF-1 $\alpha$ and lincRNA-p21 modulates the Warburg effect. Mol Cell, 2014, 53(1):88-100.

[41] Robinson EK, Covarrubias S, Carpenter S. The how and why of lncRNA function: An innate immune perspective. Biochim Biophys Acta Gene Regul Mech, 2020, 1863(4):194419.

[42] Long Y, Wang X, Youmans DT, Cech TR. How do lncRNAs regulate transcription? Sci Adv, 2017, 3(9):eaao2110.

[43] Patil DP, Chen CK, Pickering BF, Chow A, Jackson C, Guttman M, Jaffrey SR. m(6)A RNA methylation promotes XIST-mediated transcriptional repression. Nature, 2016, 537(7620): 369-373.

[44] Deng SJ, Chen HY, Ye Z, Deng SC, Zhu S, Zeng Z, He C, Liu ML, Huang K, Zhong JX, Xu FY, Li Q, Liu Y, Wang CY, Zhao G. Hypoxia-induced LncRNA-BX111 promotes metastasis and progression of pancreatic cancer through 
805 regulating ZEB1 transcription. Oncogene, 2018, 37(44): 5811-5828.

806 [45] Wang Y, Xu Z, Jiang J, Xu C, Kang J, Xiao L, Wu M, Xiong J, Guo X, Liu H. 807 Endogenous miRNA sponge lincRNA-RoR regulates Oct4, Nanog, and Sox2 in 808 human embryonic stem cell self-renewal. Dev Cell, 2013, 25(1):69-80.

809 [46] Xu J, Deng Y, Wang Y, Sun X, Chen S, Fu G. SPAG5-AS1 inhibited autophagy 810 and aggravated apoptosis of podocytes via SPAG5/AKT/mTOR pathway. Cell Prolif, $811 \quad 2020: \mathrm{e} 12738$. 


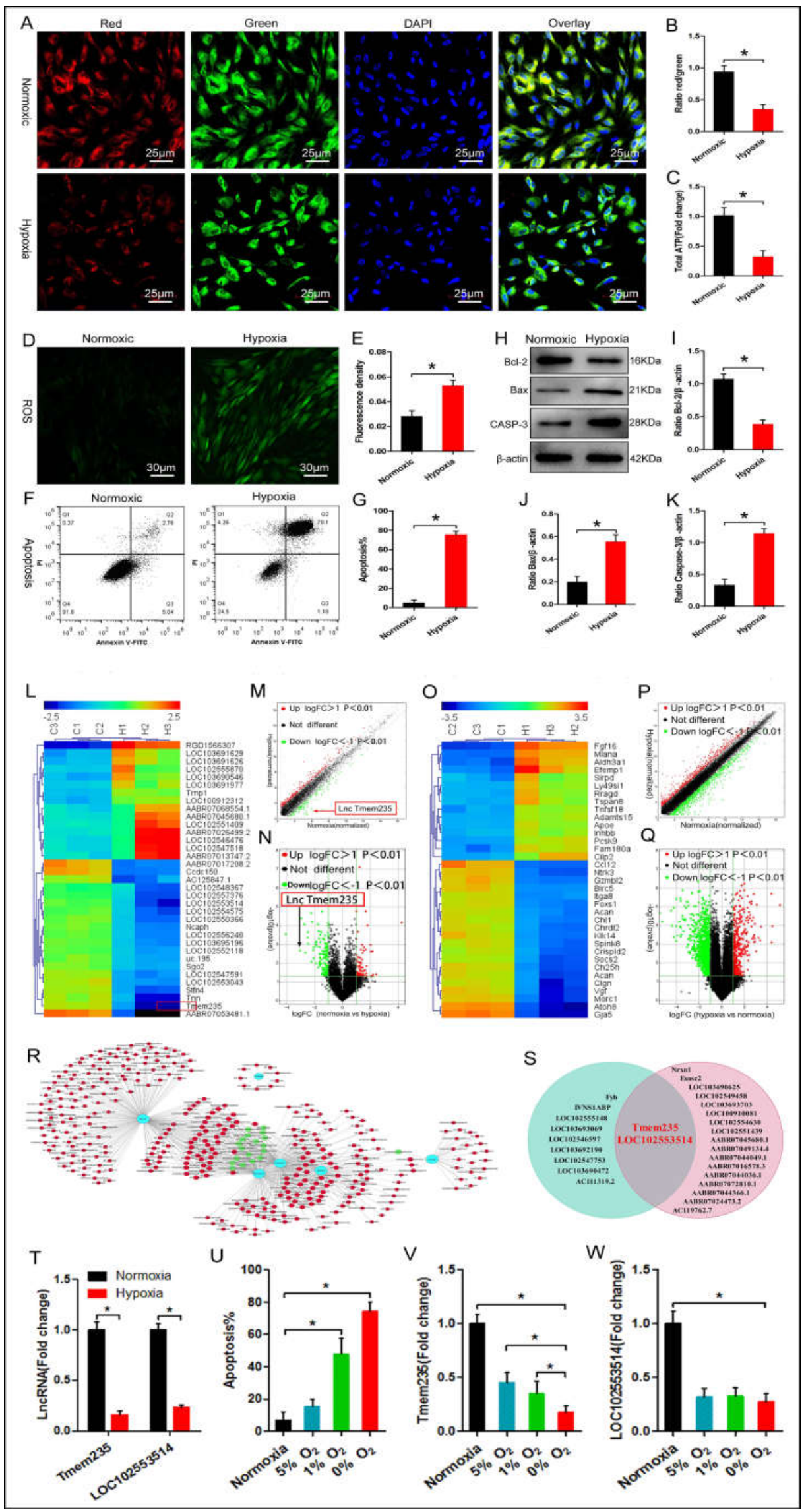


813 Figure 1. The expression of Lnc Tmem 235 is down-regulated in the process of 814 hypoxia-induced apoptosis in BMSCs: A-B. JC-1 was used to detect the 815 mitochondrial membrane potential $(n=5)$, bone-marrow mesenchymal stem cells 816 (BMSCs), 5,5',6,6' - tetrachloro - 1,1',3,3' - tetraethyl - imidacarbocyanine iodide 817 (JC-1), 4',6 - diamidino - 2 - phenylindole (DAPI); C. ATP content ( $\mathrm{n}=6)$, adenosine 818 triphosphate (ATP); D-E. The content of ROS was detected by DCFH-DA $(\mathrm{n}=5)$, reactive oxygen species (ROS), 2',7' - dichlorofluorescin diacetate (DCFH-DA); F-G.

820 Apoptosis was detected by Annexin V/PI $(n=5)$, fluoresceine isothiocyanate (FITC), 821 propidium iodide (PI); H-K. The expression levels of Bcl-2, Bax, and CASP3 were 822 analyzed by western blotting $(\mathrm{n}=3)$, B-cell lymphoma $2(\mathrm{Bcl}-2)$, Bcl-2-associated X protein (Bax), Caspase-3 (CASP-3); L. Cluster analysis of LncRNAs ( $=3)$; M.

824 Scatter plot of LncRNA expression profile, fold-change (FC); N. Volcano map of LncRNA expression profile; O. Cluster analysis of mRNAs $(n=3)$; P. Scatter plot of mRNA expression profile; Q. Volcano map of mRNA expression profile; R. 827 Co-expression analysis of mRNA-LncRNAs; S. Co-expression analysis and gene-position relationships were used to screen candidate LncRNAs; T. The expression levels of Tmem235 and LOC102553514 were verified by qPCR $(n=3)$, real-time quantitative polymerase chain reaction (qPCR); U. Apoptotic changes

831 occurred as a function of hypoxia $(n=3) ; \mathbf{V}$. Tmem235 changed with the degree of 832 hypoxia $(n=3)$; W. LOC102553514 changed with the degree of hypoxia $(n=3)$. In 833 (B, C, E, G, I-K, T-W), data are presented as means \pm standard deviations (SDs); 834 statistical significance were calculated by Student's t tests and one-way ANOVA with 835 Tukey's post-hoc tests. ${ }^{*} P<0.05$. 


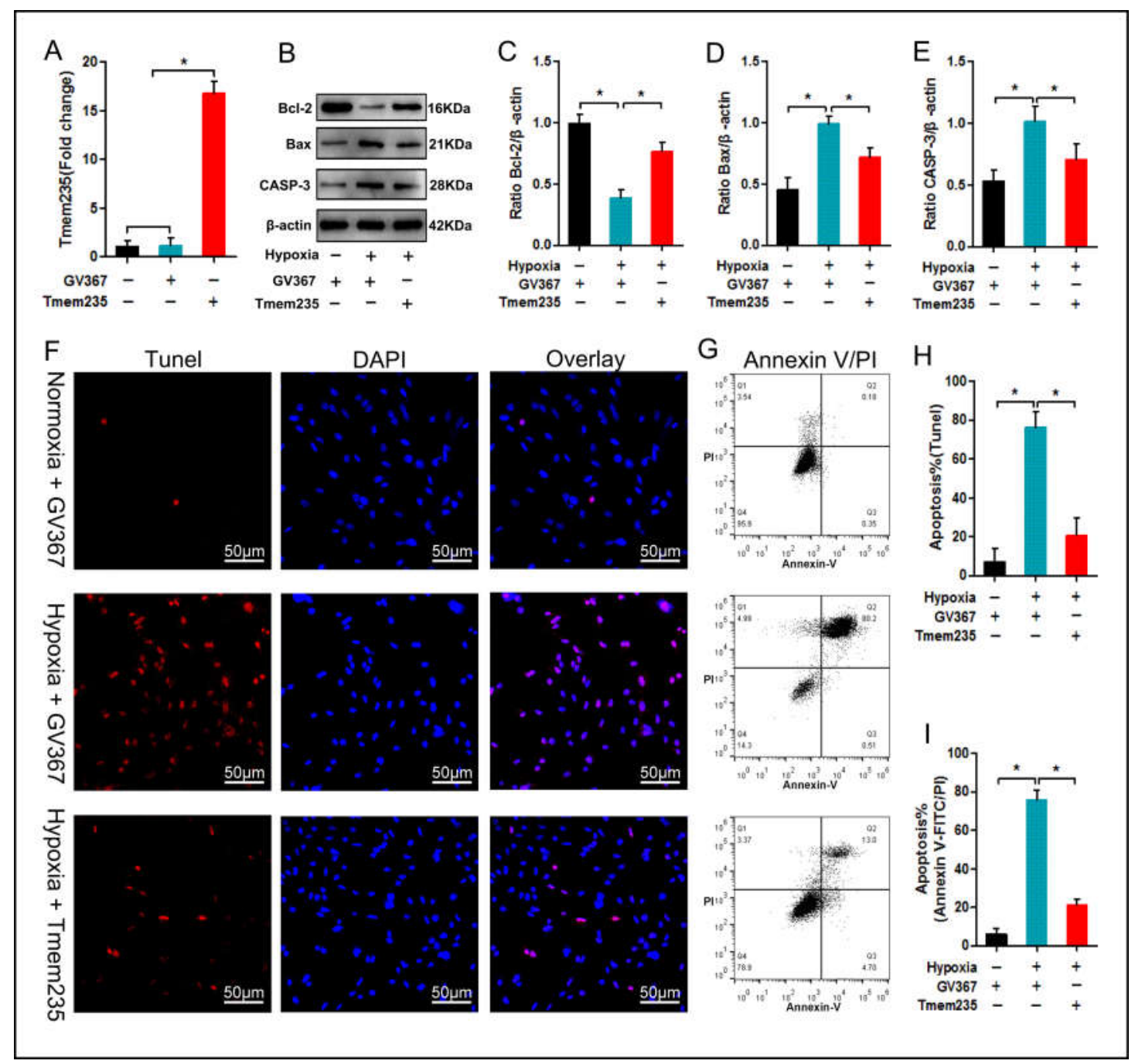

Figure 2. Lnc Tmem235 inhibits hypoxia-induced apoptosis of BMSCs: A. The expression of Lnc Tmem235 was detected by qPCR $(n=3)$, empty vector (GV367);

838 B-E. The expression levels of Bcl-2, Bax, and CASP3 were analyzed by western

839 blotting $(\mathrm{n}=3) ; \mathbf{F}$ and $\mathbf{H}$. Apoptosis was detected by Tunel/DAPI $(\mathrm{n}=5)$, terminal

840 deoxynucleotidyl transferase deoxyuridine-5'-triphosphate nick-end labeling 841 (TUNEL); G and I. Apoptosis was detected by Annexin V/PI (n = 5). In (A, C-E, $842 \mathrm{H}-\mathrm{I})$, data are presented as means \pm SDs; statistical significance was calculated by 843 one-way ANOVA with Tukey's post-hoc tests; ${ }^{*} P<0.05$. 


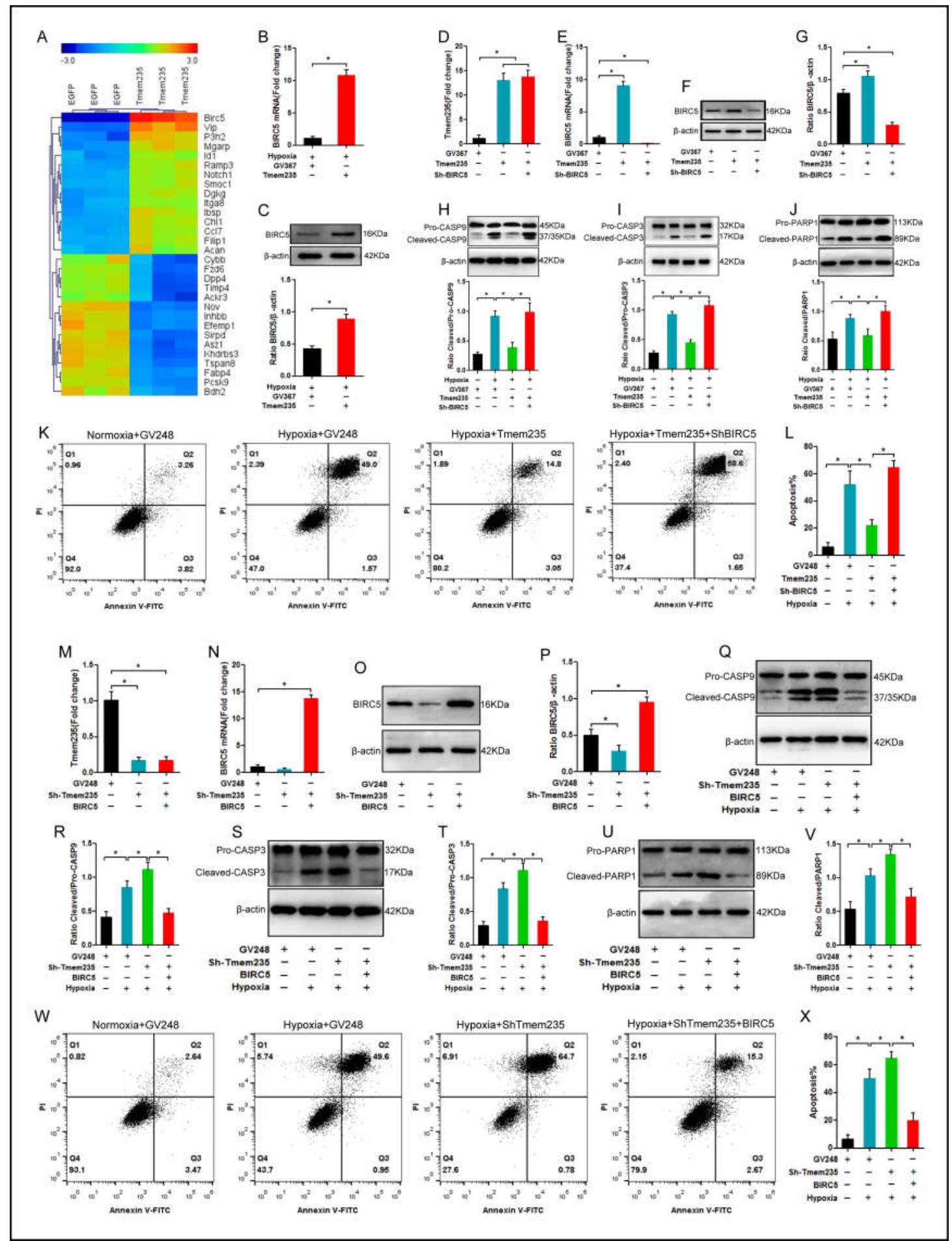

844 Figure 3. Lnc Tmem235 inhibits hypoxia-induced apoptosis of BMSCs by regulating BIRC5: A. mRNA clustering analysis $(\mathrm{n}=3)$; B. The expression of BIRC5 mRNA 846 was detected by qPCR $(\mathrm{n}=3)$, baculoviral inhibitor of apoptosis (IAP) 847 repeat-containing 5 (BIRC5); C. Western blotting was used to detect the expression of 848 BIRC5 protein $(\mathrm{n}=3)$; D. The expression of Lnc Tmem 235 was detected by qPCR ( $\mathrm{n}$ $849=3)$; E. The expression of BIRC5 mRNA was detected by qPCR $(\mathrm{n}=3) ; \mathbf{F}-\mathbf{G}$. 
850 Western blotting was used to detect the expression of BIRC5 protein $(n=3) ; \mathbf{H}$. The 851 activity of CASP-9 was analyzed by western blotting $(\mathrm{n}=3)$, Caspase-9 (CASP-9); $\mathbf{I}$. 852 The activity of CASP-3 was analyzed by western blotting $(\mathrm{n}=3)$; J. The 853 fragmentation of PARP1 was analyzed by western blotting $(\mathrm{n}=3)$, poly (ADP-ribose) 854 polymerase 1 (PARP1); K-L. Apoptosis was detected by Annexin V/PI ( $\mathrm{n}=5)$, empty 855 vector (GV248); M. The expression of Lnc Tmem235 was detected by qPCR $(\mathrm{n}=3)$; 856 N. The expression of BIRC5 mRNA was detected by qPCR $(n=3)$; $\mathbf{O}-\mathbf{P}$. The 857 expression of BIRC5 protein was detected by western blotting $(\mathrm{n}=3)$; $\mathbf{Q}-\mathbf{R}$. The 858 activity of CASP-9 was analyzed by western blotting $(\mathrm{n}=3)$; S-T. The activity of 859 CASP-3 was analyzed by western blotting $(\mathrm{n}=3)$; $\mathbf{U}-\mathbf{V}$. The fragmentation of PARP1 860 was analyzed by western blotting $(n=3) ; \mathbf{W}-\mathbf{X}$. Apoptosis was detected by Annexin 861 V/PI $(\mathrm{n}=5)$. In $(\mathbf{B}-\mathbf{E}, \mathbf{G}-\mathbf{J}, \mathbf{L}-\mathbf{N}, \mathbf{P}, \mathbf{R}, \mathbf{T}, \mathbf{V}, \mathbf{X})$, data are presented as means \pm SDs; 862 statistical significance was calculated by one-way ANOVA with Tukey's post-hoc 863 tests; ${ }^{*} P<0.05$. 


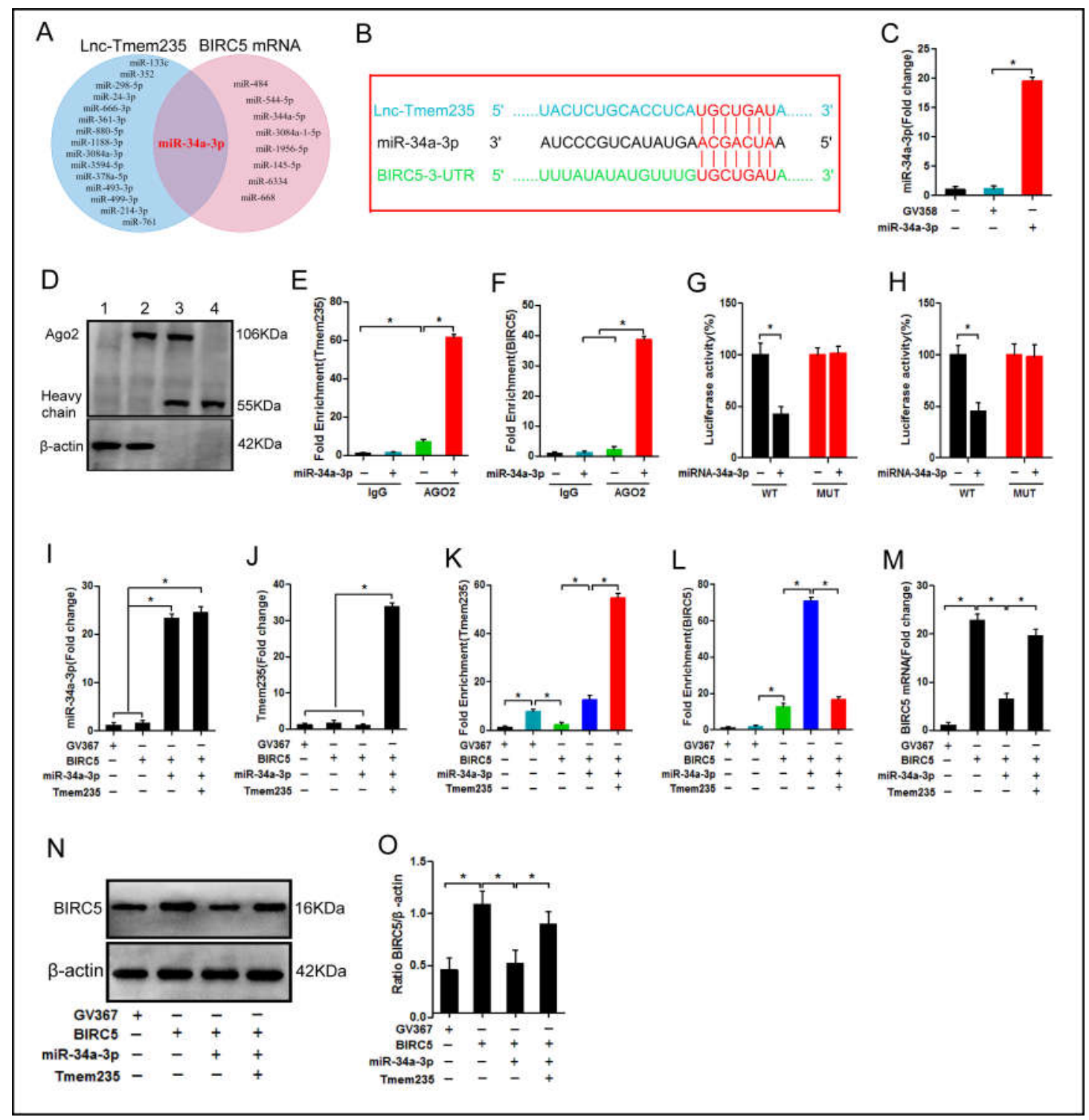

Figure 4. Lnc Tmem235 regulates BIRC5 expression by targeting miR-34a-3p: A. MiRDB and RNAhybrid predicted the miRNA of the possible combination of Lnc Tmem235 and BIRC5 mRNA 3'UTR, untranslated region (UTR), micro-RNA (miRNA); B. Binding sites of miR-34a-3p with Lnc Tmem235 and BIRC5 mRNA 3'UTR; C. The expression of miR-34a-3p was detected by qPCR ( $n=4)$, empty vector (GV358); D-F. RIP detection of enrichment of Lnc Tmem235 and BIRC5 mRNA to miRNPs $(\mathrm{n}=3)$, RNA-binding protein immunoprecipitation (RIP); G-H.

871 Luciferase-activity analysis of the binding of miR-34a-3p to Lnc Tmem235 or BIRC5 872 mRNA $(n=5)$; I. The expression of miR-34a-3p was detected by qPCR $(n=3) ; \mathbf{J}$. 873 The expression of Lnc Tmem235 was detected by qPCR $(\mathrm{n}=3)$; K. RIP detection of 874 enrichment of Lnc Tmem235 to miRNPs $(n=3)$, miRNA ribonucleoprotein 
875 complexes (miRNPs); L. RIP detection of enrichment of BIRC5 mRNA to miRNPs (n $876=3)$; M. The expression of BIRC5 mRNA was detected by qPCR $(n=3)$; $\mathbf{N}-\mathbf{O}$. 877 Western blotting was used to detect the expression of BIRC5 protein $(\mathrm{n}=3)$. In $(\mathbf{C}$, $878 \mathbf{E}-\mathbf{M}, \mathbf{O})$, data are presented as means \pm SDs; statistical significance was calculated 879 by one-way ANOVA with Tukey's post-hoc tests; ${ }^{*} P<0.05$. 


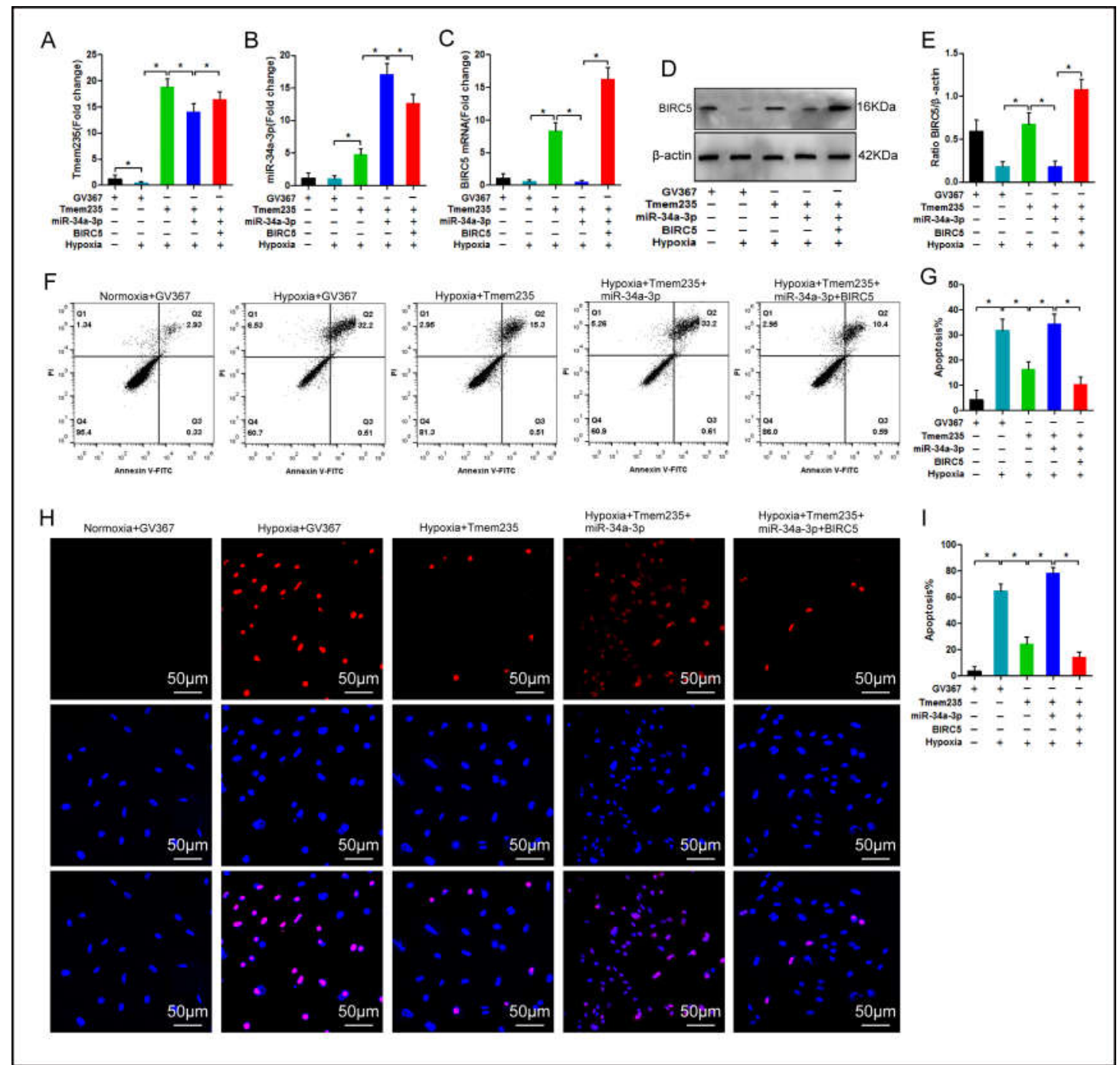

880

Figure 5. Lnc Tmem235 inhibits hypoxia-induced apoptosis of BMSCs by regulating miR-34a-3p/BIRC5: A. The expression of Lnc Tmem235 was detected by qPCR ( $\mathrm{n}=$ 4); B. The expression of miR-34a-3p was detected by $q P C R(n=4)$; C. The expression of BIRC5 mRNA was detected by qPCR $(n=4)$; D-E. Western blotting was used to detect the expression of BIRC5 protein $(\mathrm{n}=3) ; \mathbf{F}-\mathbf{G}$. Apoptosis was detected by Annexin V/PI $(\mathrm{n}=5) ; \mathbf{H}-\mathbf{I}$. Apoptosis was detected by Tunel/DAPI ( $\mathrm{n}=$ 5). In (A-C, E, G, I), data are presented as means \pm SDs; statistical significance was calculated by one-way ANOVA with Tukey's post-hoc tests; ${ }^{*} P<0.05$. 


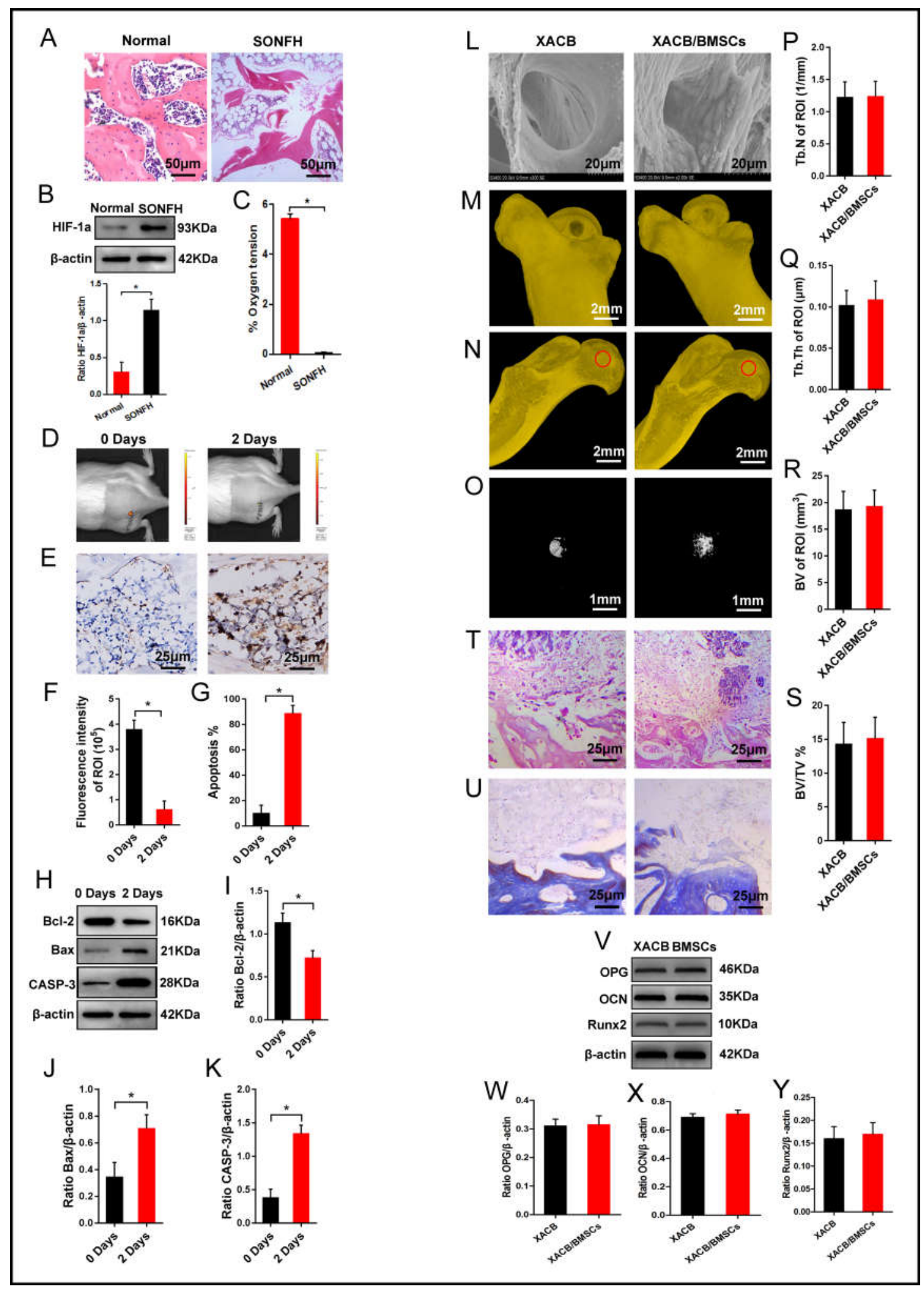

888 Figure 6. Transplantation of BMSCs to repair early SONFH: A. SONFH model (n=

889 120), steroid-induced osteonecrosis of the femoral head (SONFH); B. The expression 890 of HIF-1a in the femoral head necrotic area was detected by western blotting $(n=6)$, 891 hypoxia inducible factor 1 alpha (HIF-1a); C. Direct detection of oxygen 
concentration in the femoral head necrotic area $(n=8)$; D. At 0 days and 2 days after surgery, the DiR fluorescence intensity in the transplanted area was detected by live imaging of small animals $(\mathrm{n}=6)$; E. At 0 days and 2 days after operation, apoptosis of transplanted cells was detected by TUNEL staining $(\mathrm{n}=6) ; \mathrm{F}$. Quantitative analysis of the DiR fluorescence intensity in the transplanted area as shown in $\mathbf{D}(\mathrm{n}=6) ; \mathbf{G}$. Quantitative analysis of the proportion of TUNEL positive cells in the transplanted area as shown in $\mathbf{E}(\mathrm{n}=6) ; \mathbf{H}$. At 0 days and 2 days after surgery, the expression levels of Bcl-2, Bax, and CASP3 in the femoral head necrotic area were detected by western blotting $(\mathrm{n}=5)$; I. Quantitative analysis of Bcl-2 expression as shown in $\mathbf{H}$ ( $\mathrm{n}$ $=5)$; J. Quantitative analysis of Bax expression as shown in $\mathbf{H}(\mathrm{n}=5) ; \mathbf{K}$. Quantitative analysis of CASP-3 expression as shown in $\mathbf{H}(\mathrm{n}=5)$; L. Scanning electron microscope observation of tissue-engineered bone XACB/BMSCs $(n=8)$, xenogeneic antigen-extracted cancellous (XACB), tissue-engineered bone (XACB/ BMSCs). M-O. At 12 weeks after surgery, micro-CT analysis of the repair of the necrotic area of the femoral head $(n=6)$, red circle indicates the transplantation area; P. Quantitative analysis of the number of trabeculae as shown in $\mathbf{O}(n=6) ; \mathbf{Q}$. Quantitative analysis of the trabecular thickness as shown in $\mathbf{O}(\mathrm{n}=6) ; \mathbf{R}$. Quantitative analysis of the volume of new bone tissue as shown in $\mathbf{O}(\mathrm{n}=6)$; $\mathbf{S}$. Quantitative analysis of the volume fraction of new bone tissue as shown in $\mathbf{O}(n=6)$; T. At 12 weeks postoperatively, H\&E staining was used to evaluate the repair of the necrotic area $(n=8)$, hematoxylin-eosin $(H \& E) ; \mathbf{U}$. At 12 weeks postoperatively, Masson staining was used to evaluate the repair of the necrotic area $(n=8) ; \mathbf{V}$. At 12 weeks after surgery, the expression levels of OPG, OCN, and Runx 2 in the femoral head necrotic area were detected by western blotting $(n=5)$, osteoprotegerin $(\mathrm{OPG})$, osteocalcin (OCN), runt-related transcription factor 2 (Runx2); W. Quantitative analysis of OPG expression as shown in $\mathbf{V}(n=5)$; $\mathbf{X}$. Quantitative analysis of OCN expression as shown in $\mathbf{V}(\mathrm{n}=5)$; $\mathbf{Y}$. Quantitative analysis of Runx2 expression as shown in $\mathbf{V}(\mathrm{n}=5)$. In (B-C, F-G, I-K, P-S, W-Y), data are presented as means \pm standard deviation (SD), statistical significances were calculated by Student's t test, ${ }^{*} P<0.05$. 


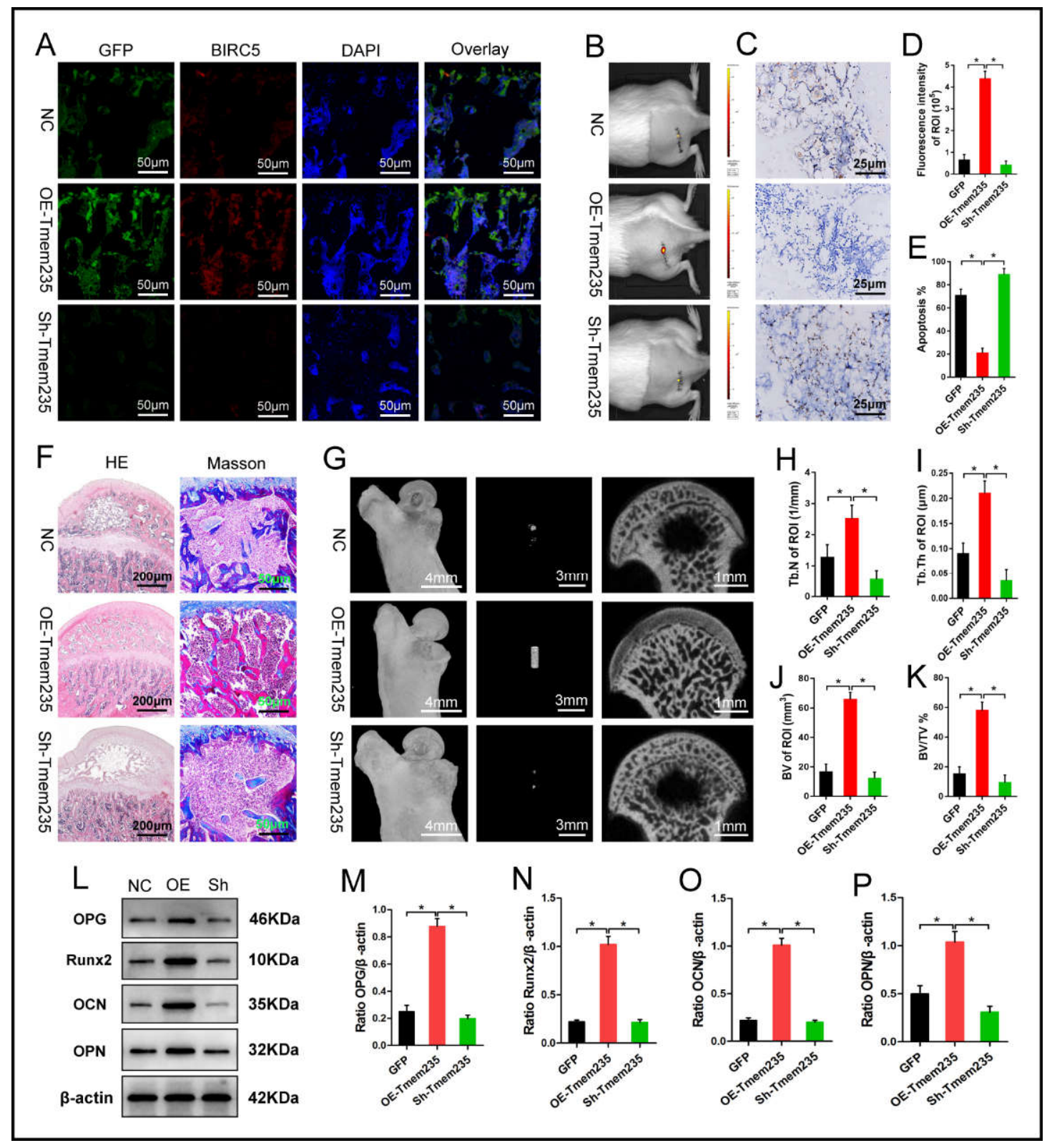

922 Figure 7. Lnc Tmem235 inhibits hypoxia-induced apoptosis of BMSCs and promotes

923 repair of early SONFH: A. At 2 days after operation, the expressions of GFP and

924 BIRC5 in the femoral head necrotic area were detected by immunofluorescence $(n=$

925 6), GFP green fluorescence labeled BMSCs, which could not penetrate bone tissue,

926 green fluorescent protein (GFP), negative control (NC), over-expression (OE), short

927 hairpin (Sh); B. At 2 days after surgery, the fluorescence intensity of DiR in the

928 transplantation area was detected by live imaging of small animals $(n=6)$, DiR red

929 fluorescence labeled BMSCs, which can penetrate bone tissue; C. At 2 days after

930 surgery, TUNEL was used to detect apoptosis in the femoral head necrotic area $(n=6)$;

931 D. Quantitative analysis of DiR fluorescence intensity in the transplanted area as 
932 shown in $\mathbf{B}(\mathrm{n}=6)$; E. Quantitative analysis of the proportion of TUNEL positive 933 cells in the transplanted area as shown in $\mathbf{C}(n=6)$; $\mathbf{F}$. At 12 weeks postoperatively, 934 H\&E staining and Masson staining were used to evaluate the repair of the necrotic

935 area $(\mathrm{n}=8)$; G. At 12 weeks after surgery, micro-CT was used to analyze the repair of necrotic area of femoral head $(n=5)$; H. Quantitative analysis of the number of trabeculae as shown in $\mathbf{G}(\mathrm{n}=5)$; I. Quantitative analysis of the trabecular thickness as shown in $\mathbf{G}(\mathrm{n}=5)$; $\mathbf{J}$. Quantitative analysis of the volume of new bone tissue as shown in $\mathbf{G}(\mathrm{n}=5)$; K. Quantitative analysis of the volume fraction of new bone tissue as shown in $\mathbf{G}(\mathrm{n}=5)$; L. At 12 weeks postoperatively, the levels of osteogenic markers (e.g., Runx2, OPN, OCN, and OPG) were detected by western blotting ( $\mathrm{n}=$ 6), osteopontin (OPN); M. Quantitative analysis of OPG expression as shown in $\mathbf{L}$ (n =6); N. Quantitative analysis of Runx2 expression as shown in $\mathbf{L}(\mathrm{n}=6) ; \mathbf{O}$. Quantitative analysis of OCN expression as shown in $\mathbf{L}(n=6) ; \mathbf{P}$. Quantitative analysis of OPN expression as shown in $\mathbf{L}(\mathrm{n}=6)$. In $(\mathbf{D}-\mathbf{E}, \mathbf{H}-\mathbf{K}, \mathbf{M}-\mathbf{P})$, data are presented as means \pm standard deviation (SD), differences are tested using one-way ANOVA with Tukey' s post hoc test, ${ }^{*} P<0.05$. 


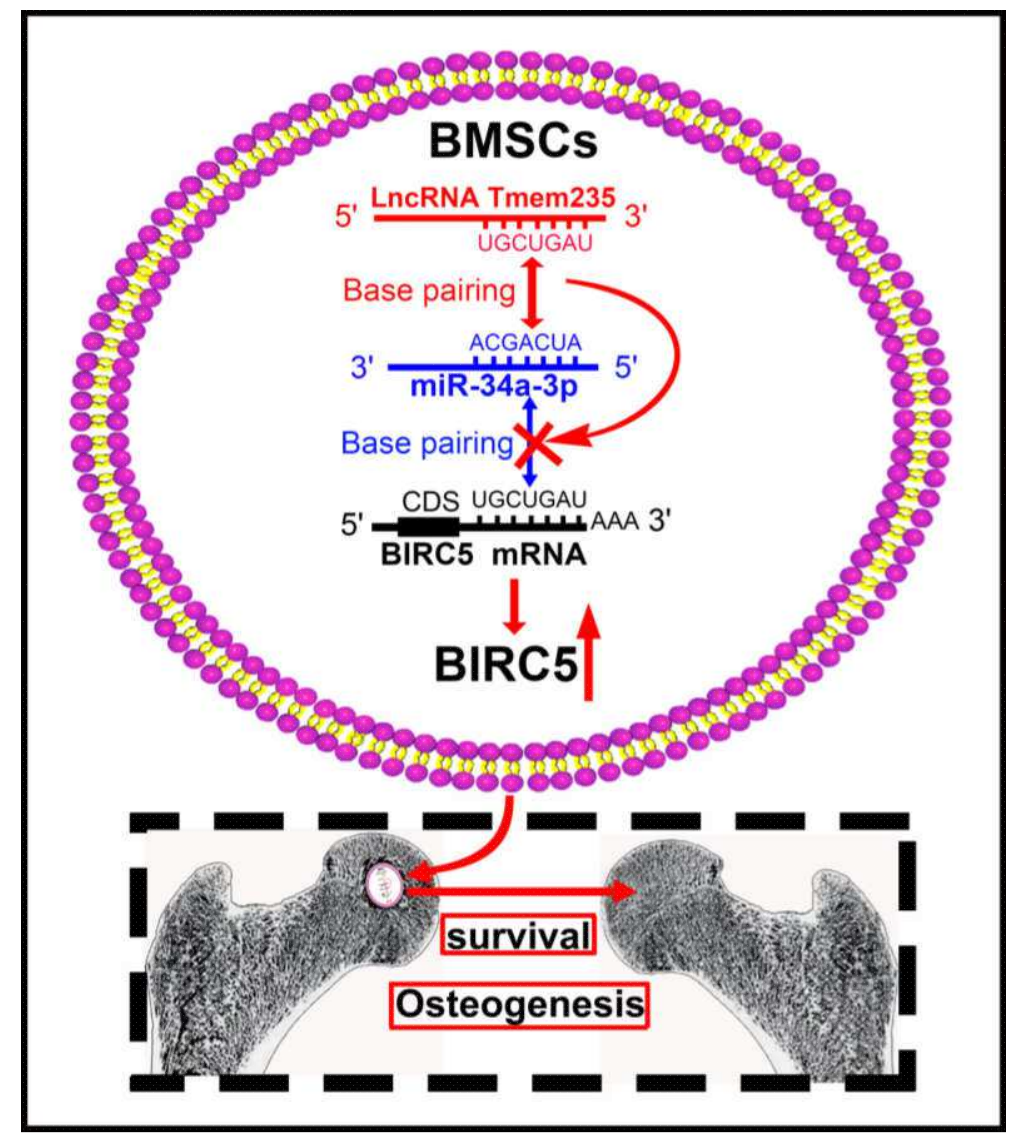

948 Figure 8. Role and mechanism LncRNA Tmem235 in SONFH: LncRNA Tmem235 949 competes with BIRC5 mRNA to bind miR-34a-3p, thereby releasing the silencing 950 effect of miR-34a-3p on BIRC5 mRNA and promoting the expression of BIRC5 951 protein; this inhibits hypoxia-induced apoptosis of BMSCs in the femoral head 952 necrotic area to ultimately improve the osteogenic repair effect of BMSCs on early 953 SONFH. 


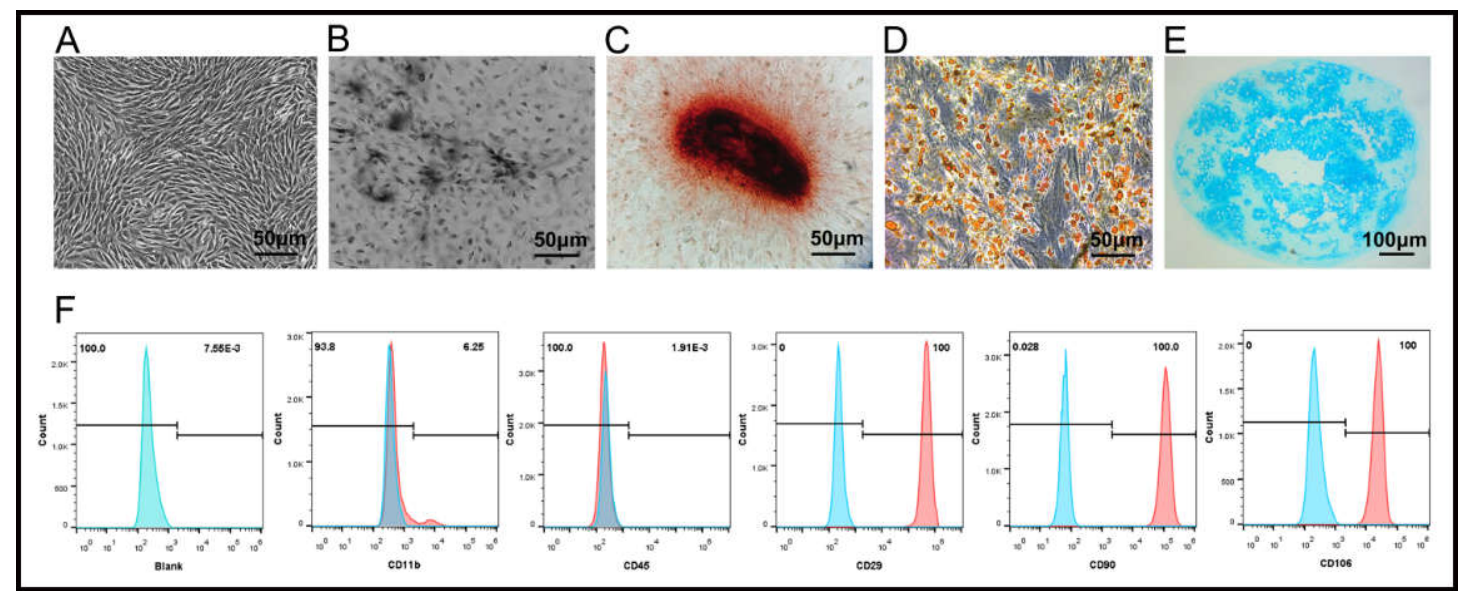

954 Supplemental Figure 1. Isolation, culture, and identification of BMSCs: A. Primary

955 BMSCs; B. Alkaline phosphatase staining after osteogenic differentiation of BMSCs

$956(\mathrm{n}=3)$; C. Alizarin-red staining after osteogenic differentiation of BMSCs $(\mathrm{n}=3)$; D.

957 Oil-red-O staining after adipogenic differentiation of BMSCs $(\mathrm{n}=3)$; E. Alisin-blue 958 staining after chondrogenic differentiation of BMSCs $(n=3)$; F. Surface antigens of 959 BMSCs - namely, CD11b, CD29, CD45, CD90, and CD106-were detected by flow 960 cytometry $(n=3)$. 


\section{Supplementary Files}

This is a list of supplementary files associated with this preprint. Click to download.

- SupplementalFig.1.jpg 